\title{
A Model for Predicting Grain Boundary Cracking in Polycrystalline Viscoplastic Materials Including Scale Effects
}

by

\author{
K. L. E. Helms and D.H. Allen \\ Aerospace Engineering Department \\ Texas A\&M University \\ College Station, TX 77843 \\ and \\ L. D. Hurtado \\ Sandia National Labs \\ Albuquerque, NM 88101
}

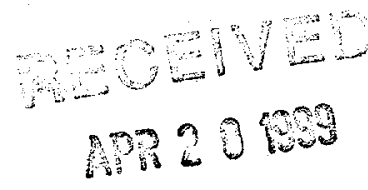

\begin{abstract}
A model is developed herein for predicting the mechanical response of inelastic crystalline solids.

Particular emphasis is given to the development of microstructural damage along grain boundaries, and the interaction of this damage with intragranular inelasticity caused by dislocation dissipation mechanisms. The model is developed within the concepts of continuum mechanics, with special emphasis on the development of internal boundaries in the continuum by utilizing a cohesive zone model based on fracture mechanics. In addition, the crystalline grains are assumed to be characterized by nonlinear viscoplastic mechanical material behavior in order to account for dislocation generation and migration. Due to the nonlinearities introduced by the crack growth and viscoplastic constitution, a numerical algorithm is utilized to solve representative problems. Implementation of the model to a finite element computational algorithm is therefore briefly described. Finally, sample calculations are presented for a polycrystalline titanium alloy with particular focus on effects of scale on the predicted response.
\end{abstract}


Key Words: polycrystalline solids, viscoplasticity, damage, cohesive zone, crack propagation, grain boundary fracture, titanium, Timetal-21S, scale effects

\section{Introduction}

Polycrystalline solids such as metals experience a variety of thermodynamically dissipative processes during inelastic deformation. These dissipative processes may include several types of dislocation rearrangement mechanisms within grains (Ashby, 1983), as well as fracture and/or sliding along grain boundaries. When these processes occur at temperatures that are generally in excess of about three-tenths of the melting point of the solid, they are inherently rate dependent. Due to the complexity of these events, and the fact that they occur in multiple grains simultaneously, analytic models of these phenomena have not been completely developed at this time.

One part of this problem that is quite difficult deals with the dissipative processes that occur along grain boundaries. Due to the migration of dislocations to grain boundaries, vacancies can open up, thereby assisting sliding and fracture between grains. In some environmental circumstances, this process can produce the majority of energy dissipation during loading. Thus, models that can physically account for this mechanism are needed.

In the current paper, the authors will discuss the deployment of a rate-dependent cohesive zone model to account for the process of grain boundary fracture and/or sliding. Inelasticity within the grains will be accounted for by employing a unified viscoplasticity model. The resulting boundary value problem will be formulated within the framework of continuum mechanics. In order to solve the resulting highly nonlinear set of integral-partial differential equations, the finite element method will be employed. 


\section{DISCLAIMER}

Portions of this document may be illegible in electronic image products. Images are produced from the best available original document. 



\subsection{Recent research}

Considerable research has focused on developing models for predicting the behavior of inelastic materials that undergo evolving damage. For purposes of the present paper, these efforts are distinguished herein as either continuum damage models, or discrete fracture models. In the continuum damage class of models, an internal variable is formulated within the macroscopic constitutive model for the multi-granular material that accounts for the locally averaged dissipation due to microfracture (Voyiadjis and Kattan, 1993). In the discrete fracture class of models, a micromechanical analysis of a multi-granular material is performed, with each microcrack modeled explicitly as an evolving internal boundary in a representative volume element (RVE) of the material. The former of these approaches has been studied in much greater detail, and due to the rather large quantity of research by this approach, no attempt will be made herein to review the works that utilize continuum damage mechanics approaches. In the current paper, the latter approach will be considered. We detail below some recent research related to this approach.

Experimental evidence indicates that numerous polycrystalline solids undergo significant grain boundary cracking, such as metals (Hertzberg, 1976; Suresh, 1991; Majumdar and Newaz, 1991), argillaceous salt (Chan et al., 1997a; Chan et al., 1997b), and sea ice (Schapery, 1997). In the current paper we have chosen to model the multi-granular behavior of Timetal-21S, a beta titanium alloy with microstructure as shown in Figure 1. Introduced in 1989, Timetal-21S was developed as a strip producible, oxidation resistant beta titanium alloy (Larsen et al., 1993). Timetal-21S is cold formable, age hardenable, and weldable. In addition to being used as a matrix material in metal matrix composites, Timetal-21S has also found limited applications in neat form. Because of many promising qualities, Timetal-21S has become the subject of much research (Foulk et al., 1998).

Numerous models have been proposed to predict the undamaged inelastic material behavior 
of Timetal-21S. At elevated temperatures, titanium is highly rate dependent. In fact, some viscoplastic theories were developed specifically for titanium-based matrices (Amold et al., 1996). However, because the Bodner-Partom (B-P) model was used herein, the discussion has been limited to the B-P viscoplastic constitutive model (Chan et al., 1988). Neu (1993) first determined the material parameters for the $\mathrm{B}-\mathrm{P}$ model in the temperature range of $23^{\circ} \mathrm{C}$ to $815^{\circ} \mathrm{C}$. Although these parameters were extremely accurate for monotonic simulations, cyclic fatigue yielded negative hardening values. The initial parameters emphasized directional hardening and upon stress reversal (compression), the directional hardening term, $Z_{D}$, and consequently the total hardening term, $Z$, became negative. Zuiker and Sanders (1995) proposed a new set of constants stressing the effects of isotropic hardening. After further review of more test data, Neu and Bodner (1995) presented a revised set of constants which also emphasized the effects of isotropic hardening. In addition, exponential evolution laws were proposed to govern the hardening rates and thermal recovery terms. A revised set of constants was developed for two specific temperatures, $482^{\circ} \mathrm{C}$ and $650^{\circ} \mathrm{C}$.

In order to model the progression of cracks in a multi-granular inelastic solid, certain pragmatic assumptions must be made in order to construct a model that is both reasonably accurate and computationally solvable. Although singular finite elements can be employed to simulate crack propagation in a general viscoplastic crystalline material, highly refined finite element meshes are required to accurately predict even the growth of a single crack. In addition, the critical energy release rate is generally not a material constant in inelastic solids. Furthermore, the geometric scale of intergranular zones is such that it is feasible to approximate them by planes of zero thickness in the undeformed state.

Cohesive zone models, first introduced by Dugdale (1960) and Barenblatt (1962), provide a computationally efficient and thermodynamically consistent method for the transition from internal 
surfaces to traction-free boundaries (Yoon and Allen, 1998; Allen and Searcy, 1998). Additionally, a cohesive zone formulation can account for a non-stationary critical energy release rate, and it does not require monitoring of the critical energy release rate in order to predict crack extension. For the above reasons, and due to the fact that numerous evolving microcracks are to be modeled simultaneously, we have chosen to employ a cohesive zone model.

Recent cohesive zone models that have influenced the current research have been proposed by Needleman (1987) and Tvergaard (1990). Needleman provided a unified framework to describe the evolution from initial debonding to complete separation in a cohesive zone using a cubic traction-displacement relation that was intended to simulate the microphysics of molecular bond stretching. Needleman has employed cohesive zones to model debonding of spherical (Needleman, 1987 ) and cylindrical (Nutt and Needleman, 1987) inclusions. Other works followed that studied the behavior of ceramic inclusions in metal matrices (Christman et al., 1989; Llorca et al., 1991), as well as modeling the decohesion of a viscoplastic block from a rigid substrate (Needleman, 1990a; Needleman, 1990b).

Tvergaard (1990) developed a model similar to Needleman's model. The formulations were identical for purely normal separation, but Tvergaard (1990) coupled normal and tangential displacements to predict other modes of separation. Tvergaard (1990) modeled the decohesion of transversely staggered $\mathrm{SiC}$ whiskers in an aluminum matrix and subsequent void formation by normal and tangential separation. Tvergaard (1995) also investigated fiber fracture and decohesion for a similar system with transversely aligned fibers. In addition to modeling metal matrix composites, Tvergaard also employed similar methods to predict crack bridging in ceramics (Tvergaard, 1992).

Several efforts have used Tvergaard's model to predict crack growth. Allen et al. (1994) 
predicted interface cracking in continuous fiber metal matrix composites. Lissenden et al. (1996) also used cohesive zone elements to predict interface cracking. Allen et al. (1996) modeled the effect of surface cracking on fiber/matrix debonding.

The cohesive zone models proposed by both Needleman and Tvergaard are elasto-plastic in the sense that they both account for dissipation in the cohesive zone that is both rate and history independent (for the case of monotonic loading). Recently the second author and coworkers have developed a cohesive zone model that can account for both history and rate dependent features of crack growth (Allen and Searcy, 1998; Yoon and Allen, 1998). It is somewhat similar to models previously developed by Schapery (1986) and Knauss and Losi (1993). This model will be utilized in the current approach.

\section{Model Development}

This section details the development of a model for predicting grain boundary cracking in polycrystalline viscoplastic solids. Towards this end, Figure 2 shows a representation of the material of interest along with a magnified view of the heterogeneous microstructure of the polycrystalline material. Both views are assumed to be sufficiently large that the concepts of continuum mechanics are applicable. We consider herein an analysis of the heterogeneous microscale. Using local continuum mechanics, we formulate the governing field equations in the following sections.

\subsection{Conservation Laws}

Consider a general continuum, $\mathrm{B}$, with initial boundary, $\partial B_{1}$, and time dependent internal boundaries, $\partial \beta_{2}(t)$, as depicted in Figure 3. The primary observable state variables to be modeled 
are the displacement, $\mathrm{u}_{\mathrm{i}}$, the Cauchy stress tensor, $\sigma_{i j}$, and the infinitesimal strain tensor, $\varepsilon_{i j}$. The conservation of mass is assumed to be trivially satisfied, assuming small displacements. The conservation of energy can be shown to be satisfied by the constitutive equations used herein, together with the assumption of constant temperature throughout the analysis (Allen, 1991). The conservation of angular momentum is satisfied by the assumption that there are no body moments when the stress tensor is symmetric. Finally, the conservation of linear momentum is satisfied by the following:

$$
\sigma_{j i, j}=0
$$

where it has been assumed that body forces and inertial effects are negligible.

\subsection{Kinematics}

Assuming small displacement gradients, the strains are defined as follows:

$$
\varepsilon_{i j} \equiv \frac{1}{2}\left(u_{i, j}+u_{j, i}\right)
$$

The above description produces strains that are an energy conjugate to the stress tensor.

\subsection{Viscoplastic Constitutive Model}

The mechanical constitutive behavior of each of the grains is assumed to be accurately modeled by a unified viscoplastic constitutive model of ordinary differential equation type. This formulation is given by (Chan, et al., 1988):

$$
\sigma_{i j}=D_{i j k l}\left(\varepsilon_{k l}-\varepsilon_{k l}^{I}\right)
$$


where $\varepsilon_{k l}^{\prime}$ is the inelastic strain tensor, and $D_{i j k l}$ is the elastic modulus tensor. The inelastic strain tensor and additional internal variables are assumed to be governed by internal variable évolution laws of the form:

$$
\dot{\alpha}_{i j}^{\eta}=\Omega_{i j}^{\eta}\left(\varepsilon_{k l}, \varepsilon_{k l}^{I}, T, \alpha_{k l}^{\mu}\right) \quad \eta=1, \ldots, n ; \mu=1, \ldots, n
$$

where $\mathrm{n}$ is the number of internal state variables. Bodner's unified viscoplasticity model (Chan et al., 1988) was chosen to model the evolution of the inelastic strain. The evolution laws are as follows:

The evolution of plastic strain (flow law):

$$
\dot{\varepsilon}_{i j}^{p}=\dot{\lambda}_{i j}, \quad \dot{\varepsilon}_{k k}^{p}=0
$$

which is related to the deviatoric stress tensor:

$$
S_{i j}=\sigma_{i j}-\frac{1}{3} \delta_{i j} \sigma_{k k}
$$

and $\dot{\lambda}$ through the kinetic equation:

$$
D_{2}^{P}=D_{0}^{P}\left\{-\left[\frac{Z^{2}}{3 J_{2}}\right]^{n}\right\}
$$


with

$$
\begin{gathered}
Z=Z_{I}+Z_{D} \\
D_{2}^{P}=\frac{1}{2} \dot{\varepsilon}_{i j^{p} \dot{E}_{i j}^{p}} \\
J_{2}=\frac{1}{2} S_{i j} S_{i j} \\
\lambda^{2}=\frac{D_{2}^{P}}{J_{2}}
\end{gathered}
$$

$\mathrm{Z}$ is the summation of isotropic and directional hardening while $\mathrm{D}_{0}$ represents the maximum shear strain rate. $J_{2}$ is a deviatoric stress invariant. The exponential behavior is governed by the kinetic parameter, $n$.

Evolution equations for the remaining internal state variables are expressed in terms of isotropic and directional hardening. The evolution law for isotropic hardening is:

$$
\dot{Z}_{I}=m_{1}\left[Z_{1}-Z_{I}\right] \dot{W}_{p}-A_{1} Z_{1}\left[\frac{Z_{I}-Z_{2}}{Z_{1}}\right]^{1}
$$


where the plastic work rate and the initial value of isotropic hardening are:

$$
\begin{gathered}
\dot{W}_{P}=\sigma_{i j} \dot{\varepsilon}_{i j}^{p} \\
Z_{\mathrm{I}}(0)=\mathrm{Z}_{0}
\end{gathered}
$$

The first term in Equation (12) involves the isotropic hardening rate term, $\mathrm{m}_{1}$, and the upper limit of isotropic hardening, $Z_{1}$. The second term contains the isotropic thermal recovery rate term $A_{1}$, the lower limit of isotropic hardening, $Z_{2}$, and the exponential term dictating isotropic thermal recovery, $r_{1}$. Directional hardening is governed by:

$$
\dot{\beta}_{i j}=m_{2}\left(Z_{3} u_{i j}-\beta_{i j}\right) \dot{W}_{p}-A_{2} Z_{1}\left[\frac{\left(\beta_{k l} \beta_{k l}\right)^{\frac{1}{2}}}{Z_{1}}\right]^{2} V_{i j}
$$

where $V_{i j}$ and $u_{i j}$ are the norms of $\beta_{i j}$ and $\sigma_{i j}$, respectively, given by:

$$
\begin{gathered}
V_{i j}=\frac{\beta_{i j}}{\left(\beta_{k l} \beta_{k l}\right)^{1 / 2}} \\
u_{i j}=\frac{\sigma_{i j}}{\left(\sigma_{k l} \sigma_{k l}\right)^{1 / 2}}
\end{gathered}
$$


Coupled with the following initial conditions:

$$
\begin{aligned}
& Z_{D}(0)=0 \\
& B_{i j}(0)=0
\end{aligned}
$$

the evolution law yields the expression for directional hardening:

$$
Z_{D}=\beta_{i j} u_{i j}
$$

The first term in Equation (15) involves the directional hardening rate term, $\mathrm{m}_{2}$, and the upper limit of isotropic hardening, $Z_{3}$. The second term contains the directional thermal recovery rate term $A_{2}$, and the exponential term governing directional thermal recovery, $\mathrm{r}_{2}$. In order to more accurately capture the behavior of Timetal-21S, Neu and Bodner (1995) wrote isotropic and directional hardening rate terms as exponential functions of the current hardening levels, $Z_{1}$ and $Z_{D}$ :

$$
\begin{gathered}
m_{1}=m_{1 b}+\left(m_{1 a}-m_{1 b}\right) \exp \left[-m_{1 c}\left(Z_{I}-Z_{o}\right)\right] \\
\left.m_{2}=m_{2 b}+\left(m_{2 a}-m_{2 b}\right) \exp \mid-m_{2 c} Z_{D}\right)
\end{gathered}
$$

where $\mathrm{m}_{1 \mathrm{a}}$ and $\mathrm{m}_{2 \mathrm{a}}$ represent initial values while $\mathrm{m}_{1 \mathrm{~b}}$ and $\mathrm{m}_{2 \mathrm{~b}}$ represent asymptotic values. In addition, the isotropic thermal recovery rate term was also rewritten as an exponential function: 


$$
A_{1}=A_{1 b}+\left(A_{1 a}-A_{1 b}\right) \exp \left[-A_{1 c}\left(Z_{I}-Z_{2}\right)\right]
$$

where $A_{1 a}$ and $A_{1 b}$ represent the initial and asymptotic values, respectively.

Implementation of Bodner's model into the finite element algorithm is most easily accomplished in the following alternate form:

$$
\dot{\sigma}_{i j}=C_{i j k l} \dot{\varepsilon}_{k l}
$$

where $\mathrm{C}_{\mathrm{ijk} k}$ is the tangent modulus tensor and $\dot{\varepsilon}_{k l}$ is the total strain rate tensor. See Allen, et al. (1994) for the derivation of the tangent modulus tensor.

\subsection{Cohesive Zone Constitutive Model}

The second author and coworkers have recently developed a micoromechanically based cohesive zone model that is based on the assumption that the intergranular material can be characterized roughly as an assemblage of parallel fibrils bridging across grains, as shown in Figure 4 . The constitutive equations for this cohesive zone model are given by (Allen and Searcy, 1998; Yoon and Allen, 1998):

$$
T_{i}(t)=\frac{u_{i}(t)}{\delta_{i} \lambda(t)}(1-\alpha(t))\left[\sigma_{i}^{f}+\int_{0}^{t} E^{c}(t-\tau) \frac{\partial \lambda}{\partial \tau} d \tau\right]
$$


where the index $i$ specifies the coordinates normal and tangential to the crack tip plane; $T_{i}$ are the corresponding components of the cohesive zone traction; $u_{i}$ are the corresponding components of the displacements; $\sigma_{i}^{f}$ is the associated stress component at the crack tip upon the initiation of damage; $\alpha$ is a damage evolution parameter describing successive fracture of the fibrils due to elongation; $\mathrm{E}^{\mathrm{C}}$ is the relaxation modulus of the cohesive zone; and $\lambda$ is the boundary imposed longitudinal strain of fibrils in the cohesive zone such that:

$$
\lambda \equiv \sqrt{\left(\frac{u_{n}}{\delta_{n}}\right)^{2}+\left(\frac{u_{t}}{\delta_{t}}\right)^{2}+\left(\frac{u_{s}}{\delta_{s}}\right)^{2}}
$$

where the subscripts $n, t$, and $s$ denote local coordinates that are normal and parallel to the cohesive zones. The empirical material length parameters, $\delta_{n}, \delta_{s}$, and $\delta_{t}$ are additional material constants for the cohesive zones. Specifically, $\delta_{n}$, is arbitrary while $\delta_{s}$ and $\delta_{t}$ are determined empirically by mixed-mode fracture experiments.

The following power law representation is used for the damage evolution:

$$
\begin{gathered}
\dot{\alpha}=\alpha_{1} \lambda^{m} \quad \dot{\lambda} \geq 0 \text { and } \alpha \leq 1 \\
\dot{\alpha}=0 \quad \dot{\lambda} \leq 0 \text { and } \alpha=1
\end{gathered}
$$

where $\alpha_{1}$ and $\mathrm{m}$ are material parameters. When $\alpha$ reaches the value of unity, the tractions go to zero and the crack propagates.

The mechanics and thermodynamics of the fracture process as predicted by the cohesive zone 
model are described in Yoon and Allen (1998). Briefly, it has been shown that the above cohesive zone model is consistent with the concepts of fracture mechanics in that there exists an available and critical energy release rate necessary for crack extension. However, in the current model, the critical energy release rate is a material property that is history and rate dependent.

\subsection{Initial and Boundary Conditions}

Both tractions and displacements are specified on the boundary of the body. Figure 5 illustrates the displacement boundary conditions imposed on the RVE. Traction boundary conditions are imposed along the cohesive zones in accordance with the equations (25) through (28).

\section{Finite Element Implementation}

The above equations have been implemented to the three-dimensional finite element code SADISTIC (Structural Analysis of Damage Induced Stresses in ThermoInelastic Composites) (Allen et al., 1994). The details of this inplementation are discussed in (Foulk et al., 1996).

\section{Model Results}

Results are presented for several trial representative volume elements (TRVE's) of Timetal-21S under monotonically increasing uniaxial displacements. The uniaxial displacement is prescribed vertically on the free end, as shown in Figure 5. In order to simplify the computations, only two dimensional plane stress analyses have been carried out. Although this is clearly an approximation, Rodin has shown in similar research using a repeating cell structure that the trends are comparable for both two- and three-dimensional analyses (1995).

Simulations of grain boundary cracking have been performed for a variety of geometries. The objective of these analyses on TRVE's is to determine if a representative volume element exists 
for this material. Herein we define a representative volume element (RVE) to be a solution for a given size of geometry in which the boundary averaged traction versus displacement relation is not significantly changed if the geometry is increased in size. Below this size, the homogenized results cannot be considered to be a representation of the macroscopic constitutive behavior of the material. Interestingly, when no damage is present, the size of this RVE is essentially infinitesimal for the material considered because without grain boundary material, the medium is spatially homogeneous. The inclusion of cohesive zones along the grain boundaries renders the material not only heterogeneous, but also, at least potentially, anisotropic due to the orientation of the crack geometry. All of this leads to the possibility that there may indeed not be a representative volume element, or equivalently, that the material considered herein may exhibit a significant size effect when viewed on any scale. The ramifications of this result would be that traditional local constitutive theory would at the very least have to be discarded in favor of a nonlocal continuum description. Therefore, this is an important issue to be considered.

In order to address the above issues, solutions have been obtained for several geometries The solution may in fact be sensitive to any or all of the following: 1) the number of grains for fixed specimen size(grain size dependence); 2) the fineness of the finite element mesh (mesh refinement); 3) the size of the representative volume element with fixed grain size (scale effect); and 4) the geometry of the grains obtained from the Voronoi tessellation model (orientation effect). Each of these effects has been studied in some detail herein. Results have been obtained for the following geometries: 5 grain model (two geometries); 10 grain model (five geometries); 30 grain model (three geometries, one with two mesh refinements); and 100 grain model (one geometry). A Voronoi tessellation approach has been used to randomly establish grain boundaries. As it is well understood that the grain structure in granular materials can be represented by Voronoi tessellation, the details 
of the technique have not been included here. The interested reader is directed to other previous studies for more details (Van der Burg and Van der Giessen, 1994). The various geometries and meshes used in this study are shown in Figures 6 and Figure 7. Statistical information about each geometry and mesh has been collected into tabular form as shown in Table 1 and Table 2, respectively.

All results herein have been obtained using the material properties shown in Table 3. Note that in order to perform these sensitivity studies the constitutive behavior in the grains has been initially assumed to be linear elastic. Although not shown, model cases have been solved for each grain geometry wherein no damage is allowed. As expected, the stresses and strains resulting from these analyses are spatially homogeneous, thus lending credence to the correctness of the geometries tested.

\subsection{Effect of Grain Size}

The effect of grain size has been simulated by $5,10,30$, and 100 grain models. Each model has varying geometry and assumes the same TRVE size of $20 \mu \mathrm{m} \times 40 \mu \mathrm{m}$ with a different number of grains thus yielding a different average grain size in each model. In the first level of analysis, one 5 grain, one 10 grain, one 30 grain, and one 100 grain model were considered. Both the length and the geometry of grain boundary cracks were observed to vary significantly in each of the models as is evidenced by the representations of crack growth for the 5 grain and 100 grain models as shown in Figures 8 and 9, respectively. A comparison of all four models resulted in predictions of force vs. prescribed displacement behavior as shown in Figure 10. From this figure, one could possibly conclude that the behavior is appearing to converge as the number of grains considered approaches 100. To confirm this conclusion, other geometries of 5 grain, 10 grain, and 30 grain models were considered as shown in Figure 11. From these simulations, one could possibly conclude that the 
behavior is converging with only 30 grains. This conclusion conflicts with the conclusion drawn from Figure 10. Consequently, we were unable to determine the effect of an average grain size from the limited cases considered.

\subsection{Effect of Finite Element Mesh Refinement}

In the geometries considered above, only coarse meshes have been used. As a finer mesh could impact convergence, two levels of mesh refinement were considered for one of the 30 grain geometries considered above. Each level of refinement approximately doubled the number of elements used. The finest mesh also incorporated multiple cohesive zone elements along the grain boundaries allowing for better ability to capture stress concentration behavior. The comparison of the predicted force vs. prescribed displacement for the three different meshes is shown in Figure 12. Although each of the finer meshes more smoothly captured the damage accumulation, there was no marked difference in overall response thus leading to the conclusion that mesh refinement alone may not have a significant effect on convergence.

\subsection{Scale Effect}

As all of the TRVE's considered above were the same size, two different size TRVE's were extracted from the 100 grain geometry to allow for study of scale effect on convergence. The first TRVE extracted contained a 10 grain $5 \mu \mathrm{m} \times 10 \mu \mathrm{m}$ portion from the lower left corner of the 100 grain geometry. The second TRVE extracted was larger containing a 30 grain $10 \mu \mathrm{m} \times 20 \mu \mathrm{m}$ portion which included all of the $5 \mu \mathrm{m} \times 10 \mu \mathrm{m}$ TRVE. The response of these two simulations are compared to the original $10 \mu \mathrm{m} \times 20 \mu \mathrm{m} 100$ grain model in Figure 13. Although the predicted responses did exhibit a noticeable trend in strength, larger TRVE's must be considered to identify convergence.

\subsection{Orientation Effect}

To simulate the effect of orientation of grains on predicted response, the grain size was kept constant 
while various geometries were considered. The response of four different geometries of a $10 \mu \mathrm{mx}$ $20 \mu \mathrm{m}$ TRVE each with 10 grains is shown in Figure 14. The responses show a great deal of variation leading to the conclusion that orientation has a significant effect on thepredicted response of the TRVE.

\section{Conclusion}

A model to predict grain boundary cracking has been presented and applied to a general polycrystalline viscoplastic material, Timetal-21S, a titanium alloy. This model has incorporated both the effects of material inelasticity and damage. Material inelasticity has been predicted using Bodner's unified viscoplastic constitutive model. Damage has been included explicitly via cohesive zone elements.

Previous studies have shown that both inelastic material behavior which is rate dependent and damage effects must be incorporated into effective modeling of typical polycrystalline materials such as titanium, argillaceous salts, or sea ice. The current modeling efforts focused on determining a suitable representative volume element (if one exists) by considering the separate effects of 1) grain size, 2) mesh refinement, 3) scale, and 4) orientation. To this end, several geometries were considered to isolate these individual parameters. Average grain size alone was not found to lead towards convergent behavior. Using the one trial representative volume element considered, mesh refinement by itself was found to have only a small effect on predicted response. The scale parameter alone was found to affect predicted material response; however, for the three TRVE's considered, no trend towards convergent behavior was observed. Varying only orientation was found to significantly affect material response, and further study is warranted to better describe this effect. No attempt in the current study, however, was made to fully vary the other three parameters simultaneously; consequently, a definitive conclusion regarding convergence or lack of 
convergence to determine a RVE could not be drawn.

\section{Acknowledgments}

The authors gratefully acknowledge the support provided for this research by Sandia National Laboratories under contract number AT-8781. Sandia is a multiprogram laboratory operated by Sandia Corporation, a Lockheed Martin Company, for the United States Department of Energy under Contract DE-AC04-94AL85000. We also wish to thank Jill Scheidt, Kyle Clayton, Chad Searcy, Ross Brown, Gary Seidel, Virginia Oxford, Lisa Biggs, and Annilee Travers for their assistance with mesh generation and Todd Rinaldo for assistance with crack animation software.

\section{References}

D. H. Allen, "Thermomechanical Coupling in Inelastic Solids," Applied Mechanics Review 8 (1991) $361-373$

D.H. Allen, R. H. Jones, and J. G. Boyd, "Micromechanical Analysis of a Continuous Fiber Metal Matrix Composite Including the Effects of Matrix Viscoplasticity and Evolving Damage,"Journal of Mechanics and Physics of Solids, Vol. 42, No. 3, (1994) 502-529.

D.H. Allen, J.W. Foulk, and K.L.E. Helms, "A Model for Predicting the Effect of Environmental Degradation on Damage Evolution in Metal Matrix Composites," Applications of Continuum Damage Mechanics to Fatigue and Fracture, Orlando, FL (1996).

D. H. Allen and C. R. Searcy, "A Micromechanical Model for Viscoelastic Cohesive Zones," submitted to International Journal of Fracture (1998). 
S. M. Arnold, A. F. Saleeb, and M. G. Castelli, “A Fully Associative, Nonlinear, Kinematic, Unified Viscoplastic Model for Titanium-Based Matrices," Life Prediction Methodology for Titanium Matrix Composites, ASTM STP 1253 (eds. W. S. Johnson, J. M. Larsen, and B. N. Cos) (1996) 231-256.

M. F. Ashby, "Mechanisms of Deformation and Fracture," Advances in Applied Mechanics 23 (1983) $117-177$.

G. I. Barenblatt, "The Mathematical Theory of Equilibrium Cracks in Brittle Fracture," Advances in Applied Mechanics 7 (1962) 55-129.

K.S. Chan, S.R. Bodner, and U.S. Lindholm, " Phenomenalogical Modeling of Hardening and Thermal Recovery in Metals," Journal of Engineering Materials and Technology, (1988). 1-8.

K. S. Chan, S. R. Bodner, A. F. Fossum, D. E. Munson, "A Damage Mechanics Treatment of Creep Failure in Rock Salt," International Journal of Damage Mechanics 6 (1997) 121-152.

K. S. Chan, N. S. Brodsky, A. F. Fossum, D. E. Munson, and S. R. Bodner, "Creep-Induced Cleavage Fracture in WIPP Salt Under Indirect Tension," Journal of Engineering Materials and Technology 119 (1997) 393-400.

T. Christman, A. Needleman, and S. Suresh, "An Experimental and Numerical Study of Deformation in Metal-Ceramic Composites," Acta Metallurgica, Vol. 37, No. 11, (1989) 3029-3050. 
D. S. Dugdale, "Yielding of Steel Sheets Containing Slits," Journal of the Mechanics and Physics of Solids 8 (1960) 100-104.

J.W. Foulk, K.L.E. Helms, and D.H. Allen, "Life Prediction in Continuous Fiber Metal Matrix Composites Subjected to Environmental Degradation," proceedings of the 11 th Technical Conference of the American Society for Composites, Atlanta, GA, October 7-9 (1996).

J. W. Foulk, D. H. Allen, and K. L. E. Helms, "A Model for Predicting the Damage and Environmental Degradation Dependent Life of SCS-6/Timetal 21S.[0] $]_{4}$ Metal Matrix Composite," to appear in Mechanics \& Materials (1998).

R. W. Hertzberg, Deformation and Fracture Mechanics of Engineering Materials, John Wiley \& Sons (1976).

W. G. Knauss and G. U. Losi, "Crack Propagation in a Nonlinearly Viscoelastic Solid with Relevance to Adhesive Bond Failure," Journal of Applied Mechanics 60 (1993) 793-801.

J.M. Larsen, S.M. Russ, and J.W. Jones, "Possibilities and Pitfalls in Aerospace Applications of Titanium Matrix Composites," in the proceedings of the NATO AGARD Conference on Characterization of Fibre Reinforced Titanium Metal Matrix Composites, Bordeaux, France(1993).

C.J. Lissenden, C.T. Herakovich, and M. Pindera, "Inelastic Deformation of Titanium Matrix 
Composites Under Multiaxial Loading," Life Prediction Methodology for Titanium Matrix Composites, ASTM STP 1253, W.S. Johnson, J.M. Larsen, and B.N. Cox, Eds., ASTM (1996) 257277.

L. Llorca, A. Needleman, S. Suresh, "An Analysis of the Effects of Matrix Void Growth on Deformation and Ductility in Metal-Ceramic Composites," Acta Metallurgica et Materialia, Vol. 39, No. 10 (1991) 2317-2335.

B. S. Majumdar and G. M. Newaz, "Thermomechanical Fatigue of a Quasi-Isotropic Metal Matrix Composite," Composite Materials: Fatigue and Fracture (Third Volume), ASTM STP 11 (ed. T. K. O'Brien) (1991) 732-752.

A. Needleman, "A Continuum Model for Void Nucleation by Inclusion Debonding," Journal of Applied Mechanics, 54 (1987) 525-531.

A. Needleman, "An Analysis of Tensile Decohesion Along A Interface," Joumal of Mechanics and Physics of Solids, Vol. 38, No. 3, (1990) 289-324.

A. Needleman, "An Analysis of Decohesion Along an Imperfect Interface," International Journal of Fracture, Vol. 42, (1990) 21-40.

R.W. Neu, "Nonisothermal Material Parameters for the Bodner-Partom Model," in the proceedings of the ASME Winter Annual Meeting, New Orleans, LA (1993). 
R.W. Neu, and S.R. Bodner, "Determination of the Material Constants of Timetal 21S for a Constitutive Model," internal report prepared for Wright Laboratory Materials Directorate, WrightPatterson AFB, OH (1995).

S.R. Nutt, and A. Needleman, "Void Nucleation at Fiber Ends in Al-SiC Composites," Scripta Metallurgica, Vol. 21, (1987) 705-710.

G. J. Rodin, "Stress Transmission in Polycrystals With Frictionless Grain Boundaries," Joumnal of Applied Mechanics 62 (1995) 1-6.

R. A. Schapery, "A Micromechanical Model for Non-Linear Viscoelastic Behavior of ParticleReinforced Rubber with Distributed Damage, Engineering Fracture Mechanics 25 (1986) 845-867.

R. A. Schapery, "Thermoviscoelastic Constitutive Equations for Polycrystalline Ice," ASCE Journal of Cold Regions Engineering 2 (1997), 146-157.

S. Suresh, Fatigue of Materials, Cambridge University Press (1991).

V. Tvergaard, "Effect of Fibre Debonding in a Whisker-reinforced Metal" Materials Science and Engineering A125 (1990) 203-213.

V. Tvergaard, "Effect of Ductile Particle Debonding During Crack Bridging in Ceramics," 
International Joumal of Mechanical Science, Vol. 34, No. 8, (1992) 635-649.

V. Tvergaard, "Fibre Debonding and Breakage in a Whisker-Reinforced Metal," Materials Science and Engineering, Vol, A190, (1995) 215-222.

M.W.D. Van der Burg and E. Van der Giessen, "Simulation of Microcrack Propagation in Creeping Polycrystals Due to Diffusive Grain Boundary Cavitation," Applied Mechanics Review 47 (1994) $122-131$.

G. Z. Voyiadjis and P. I. Kattan, "Local Approach to Damage in Elasto-Plastic Metal Matrix Composites," International Journal of Damage Mechanics 2 (1993), 96-114.

C. Yoon and D. H. Allen, "Damage Dependent Constitutive Behavior and Energy Release Rate for a Cohesive Zone in a Thermoviscoelastic Solid," submitted to International Journal of Fracture (1998).

J.R. Zuiker and B.P. Sanders, "Analysis of Pinned Joints in Viscoplastic Monolithic Plates," Numerical Implementation and Application of Constitutive Models in the Finite Element Method, J.A. Sherwood, ed., American Society of Mechanical Engineers - Applied Materials Division, New York (1995)37-48. 


\section{Figure List}

Figure 1 Multi-grain microstructure of Timetal 21S.

Figure 2 Continuum representation of a polycrystalline material.

Figure 3 A general body, $\beta$, with initial boundary, $\partial \beta_{1}$, and time dependent internal boundaries, $\partial \beta_{2}(t)$.

Figure 4 Depiction of assemblage of parallel fibrils bridging across grain boundaries.

Figure 5 Boundary conditions imposed on representative volume element.

Figure 6a Geometry 1: five grain Voronoi Tessellation $20 \mu \mathrm{m} \times 40 \mu \mathrm{m}$ trial representative volume element.

Figure $6 \mathrm{~b} \quad$ Geometry 2: five grain Voronoi Tessellation $20 \mu \mathrm{m} \times 40 \mu \mathrm{m}$ trial representative volume element.

Figure 6c Geometry 3: ten grain Voronoi Tessellation $20 \mu \mathrm{m} \times 40 \mu \mathrm{m}$ trial representative volume element.

Figure 6d Geometry 4: ten grain Voronoi Tessellation $20 \mu \mathrm{m} \times 40 \mu \mathrm{m}$ trial representative volume element.

Figure 6e Geometry 5: ten grain Voronoi Tessellation $20 \mu \mathrm{m} \times 40 \mu \mathrm{m}$ trial representative volume element.

Figure 6f Geometry 6: ten grain Voronoi Tessellation $20 \mu \mathrm{m} \times 40 \mu \mathrm{m}$ trial representative volume element.

Figure 6g Geometry 7: ten grain Voronoi Tessellation (part of Geometry 11) $5 \mu \mathrm{m} \times 10 \mu \mathrm{m}$ trial representative volume element. 
Figure 6h Geometry 8: thirty grain Voronoi Tessellation $20 \mu \mathrm{m} \times 40 \mu \mathrm{m}$ trial representative volume element.

Figure 6i Geometry 9: thity grain Voronoi Tessellation $20 \mu \mathrm{m} \times 40 \mu \mathrm{m}$ trial representative volume element.

Figure 6j Geometry 10: thirty grain Voronoi Tessellation (part of Geometry 11) 10 $\mu \mathrm{m} \times 20 \mu \mathrm{m}$ trial representative volume element.

Figure 6k Geometry 11: one hundred grain Voronoi Tessellation $20 \mu \mathrm{m} \times 40 \mu \mathrm{m}$ trial representative volume element.

Figure7a Mesh 1: Geometry 1 (five grain) with 16 elements.

Figure 7b Mesh 2: Geometry 3 (ten grain) with 28 elements.

Figure 7c Mesh 3: Geometry 8 (thirty grain) with 97 elements

Figure 7d Mesh 4: Geometry 11 (one hundred grain) with 292 elements

Figure 7e Mesh 5: Geometry 2 (five grain) with 26 elements.

Figure 7f Mesh 6: Geometry 4 (ten grain) with 37 elements.

Figure 7g Mesh 7: Geometry 5 (ten grain) with 37 elements.

Figure 7h Mesh 8: Geometry 6 (ten grain) with 44 elements.

Figure 7i Mesh 9: Geometry 9 (thirty grain) with 109 elements.

Figure $7 \mathrm{j} . \quad$ Mesh 10: Geometry 8 (thirty grain) with 185 elements.

Figure 7k Mesh 11: Geometry8 (thirty grain) with 290 elements.

Figure $71 \quad$ Mesh 12: Geometry 7 (ten grain) with 37 elements.

Figure $7 \mathrm{~m}$ Mesh 13: Geometry 10 (thirty grain) with 88 elements.

Figure $8 \quad$ Predicted grain boundary crack growth for a 5 grain model (Mesh 1)

Figure 9 Predicted grain boundary crack growth for a 100 grain model (Mesh 4) 
Figure 10 Predicted force vs. displacement by average grain size for $5,10,30$, and 100 grain models (Mesh 1, Mesh 2, Mesh 3, and Mesh 4)

Figure 11 Predicted force vs. displacement by average grain size for 5, 10, and 30 grain models (Mesh 5, Mesh 6, Mesh 7, Mesh 8, and Mesh 9)

Figure 12 Predicted force vs. displacement for mesh refinement of a 30 grain model (Mesh 3, Mesh 10, and Mesh 11)

Figure 13 Predicted force vs. displacement by trial representative volume element size for 10, 30, and 100 grain models (Mesh 4, Mesh 12, and Mesh 13)

Figure 14 Predicted force vs. displacement for 10 grain models by for orientations (geometries) (Mesh 2, Mesh 6, Mesh 7, and Mesh 8) 
9. List of Tables

Table $1 \quad$ Geometries Considered

Table 2 Meshes Considered

Table 3 Material Properties for Timetal $21 \mathrm{~S}$ at $482{ }^{\circ} \mathrm{C}$ 
Figure 1

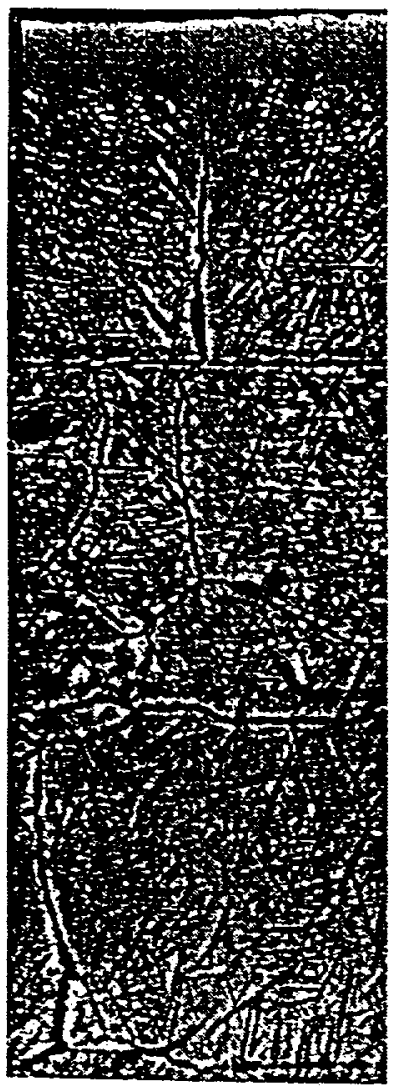


Figure 2

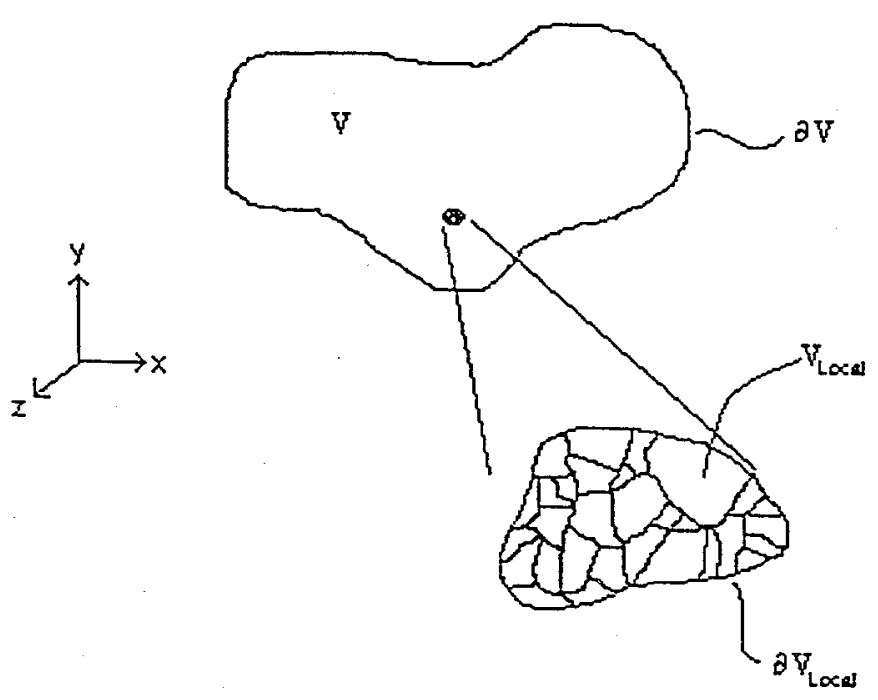


Figure 3

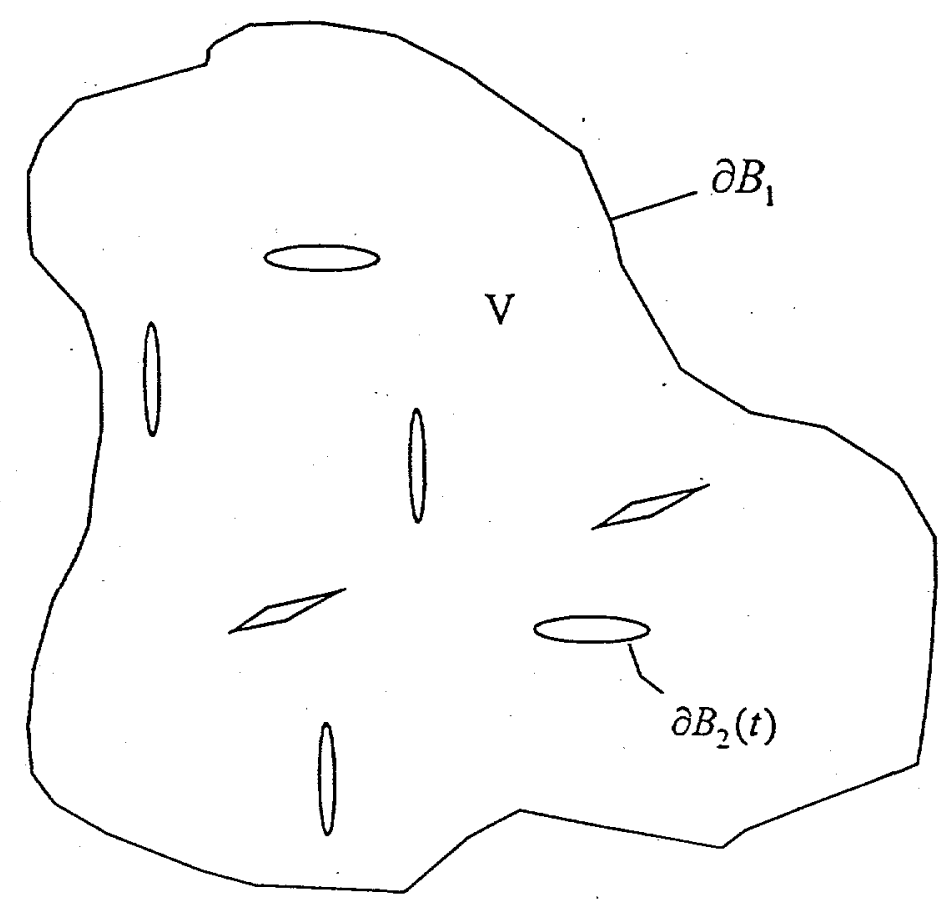


Figure 4

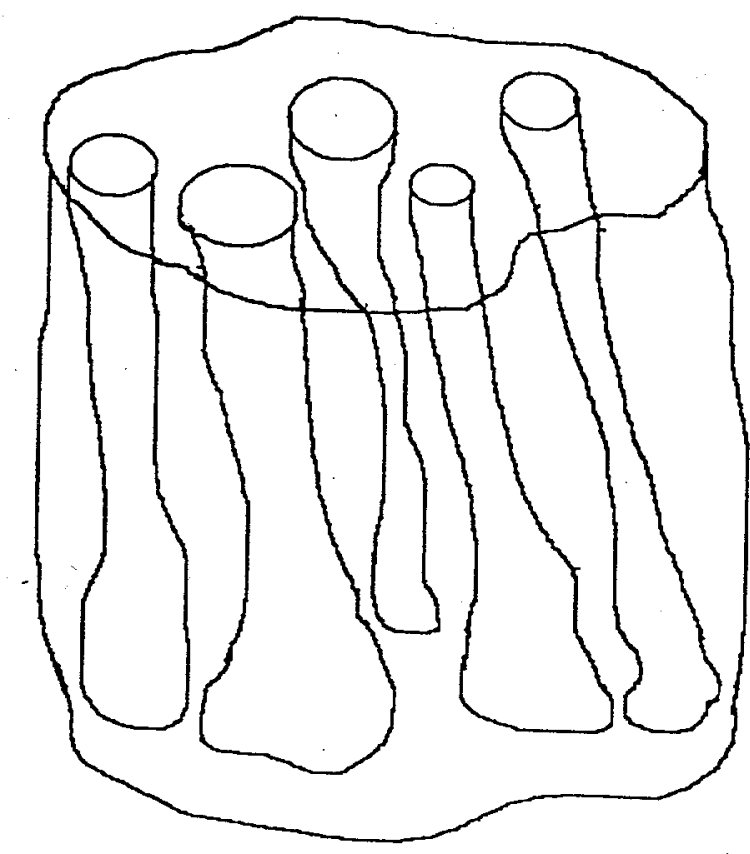


Figure 5

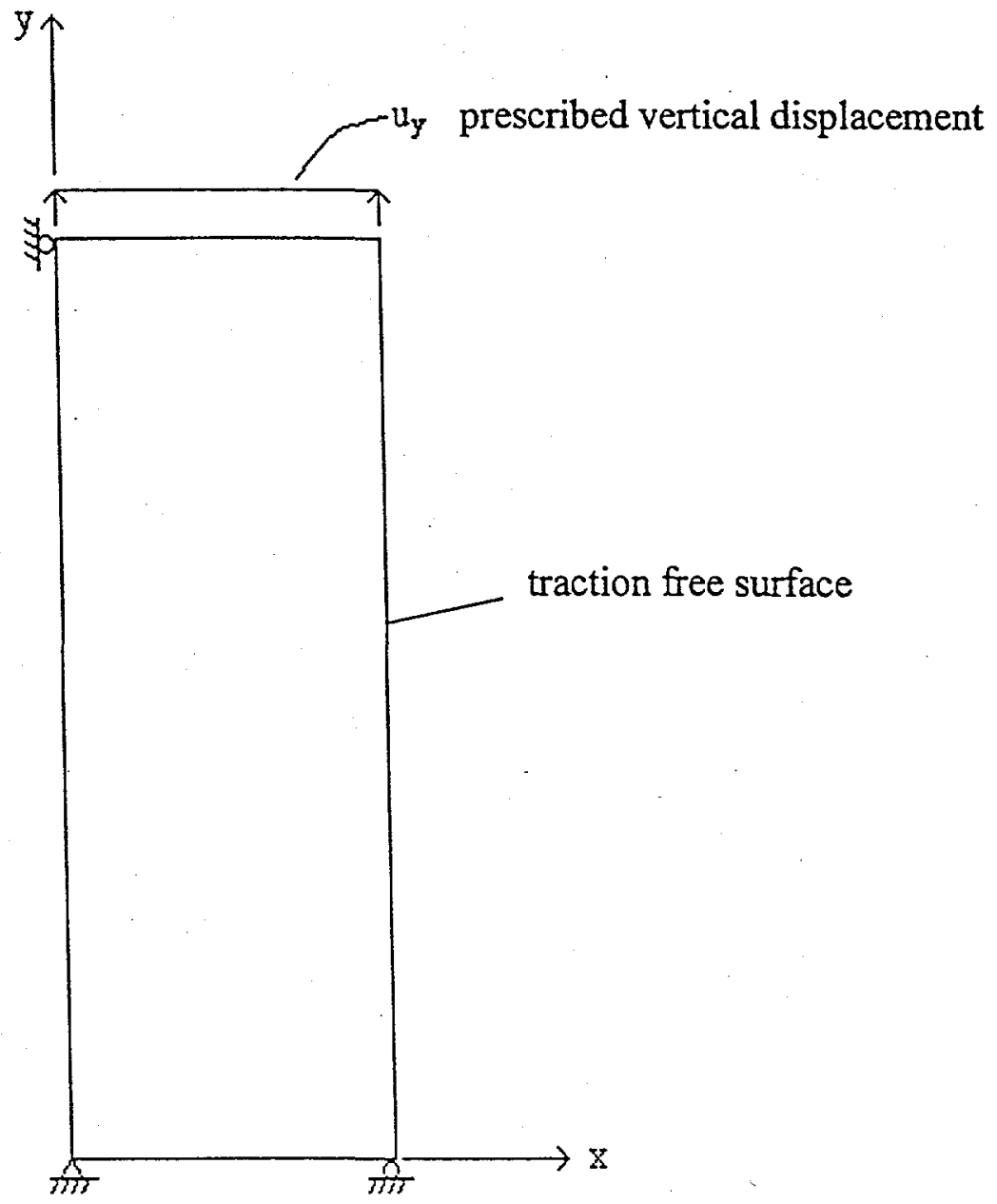

Rollers and pin supports indicate: along $x=0, u_{x}=0$ and along $y=0, u_{y}=0$ 
Figure 6a

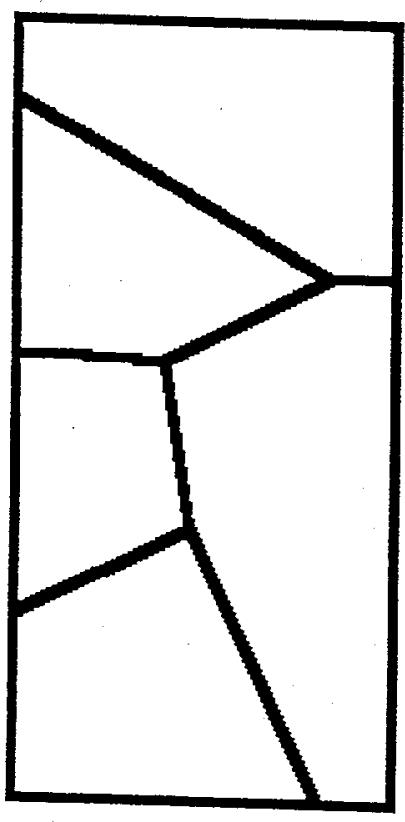




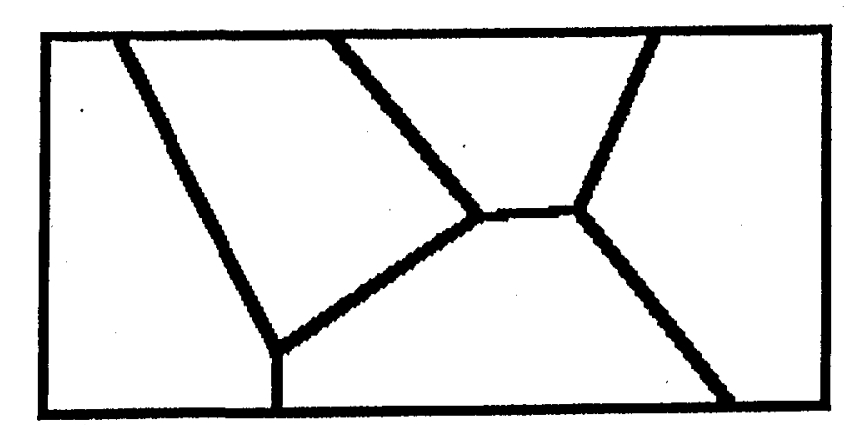


Figure 6c

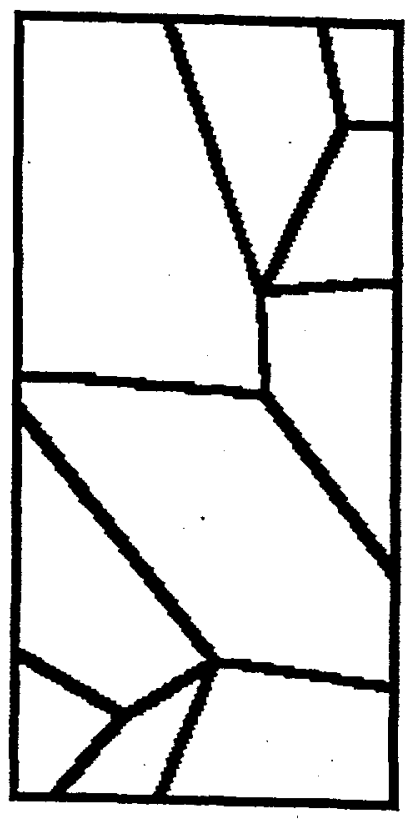


$n$
$n$
0
0
0
0
2

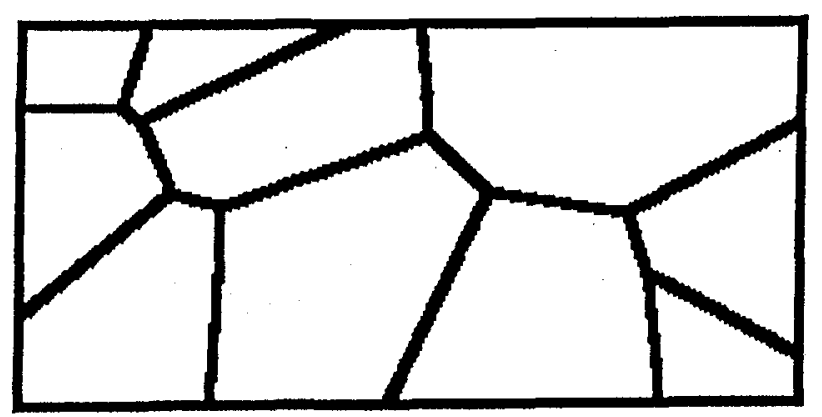


Figure 6e

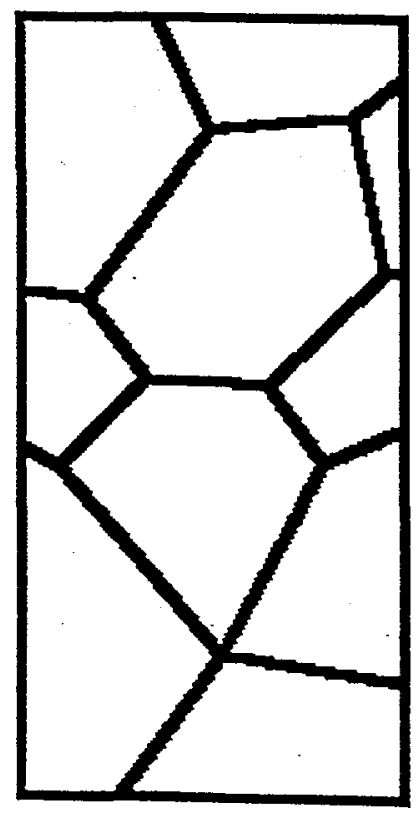


fit 
国 
Figure $6 \mathrm{~h}$

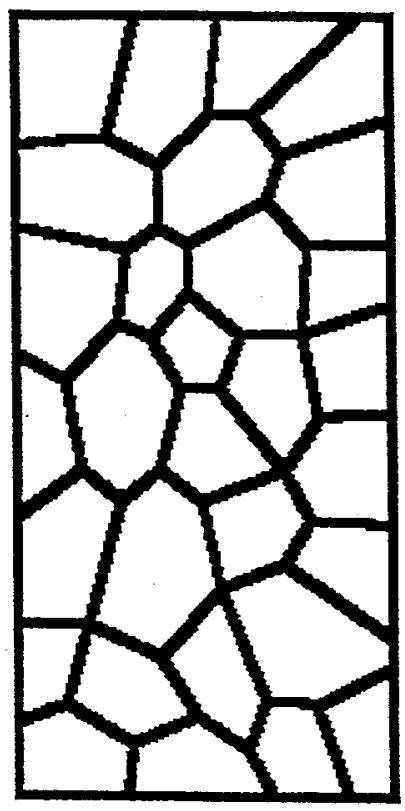


Figure 6i

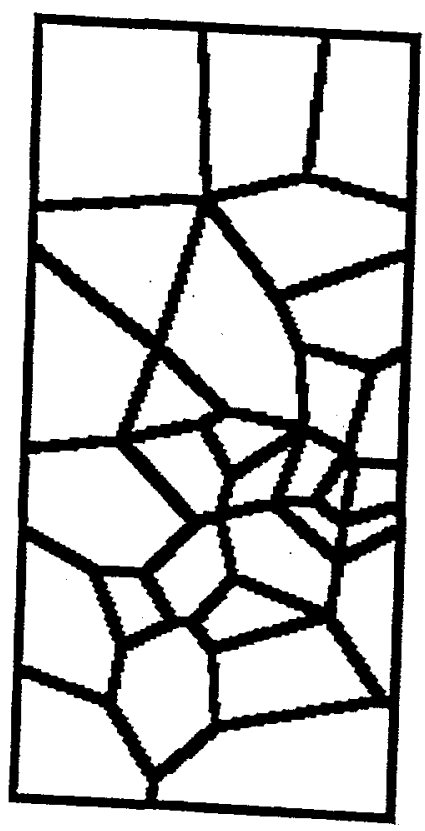


Figure 6j

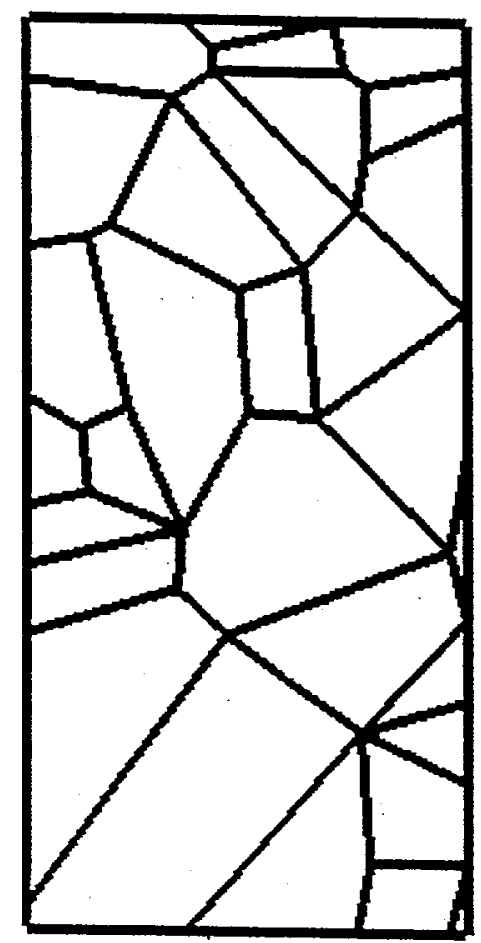


Figure 6k

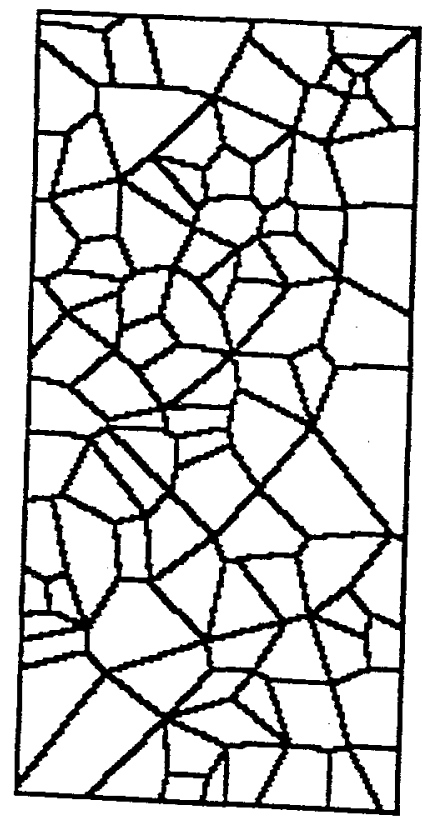


Figure 7a

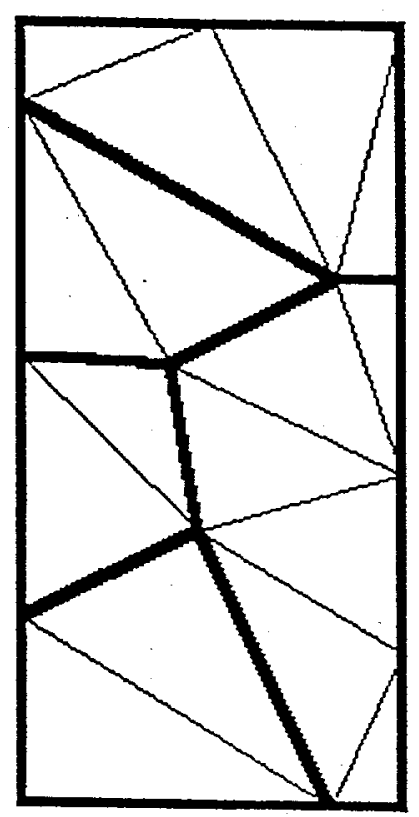


Figure $7 b$

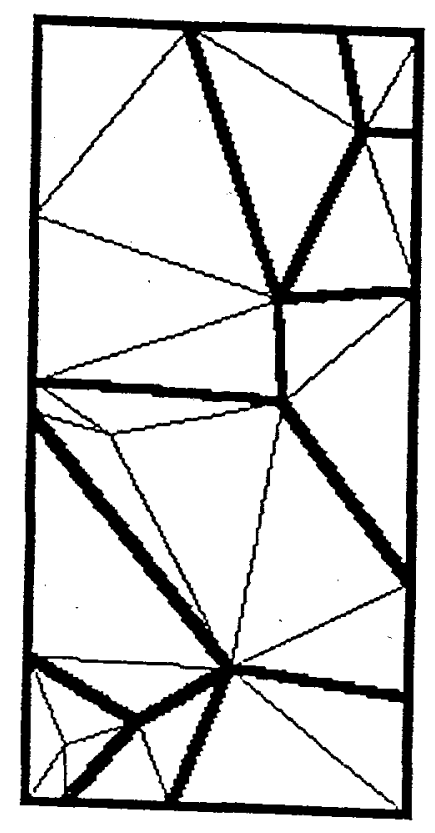


Figure $7 d$

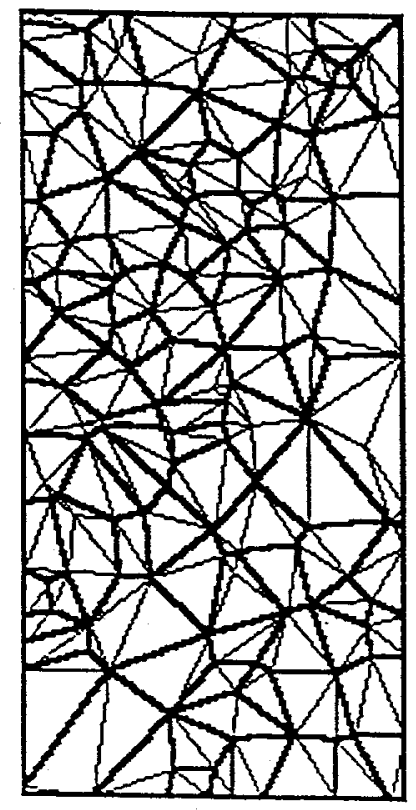


Figure $7 \mathrm{c}$

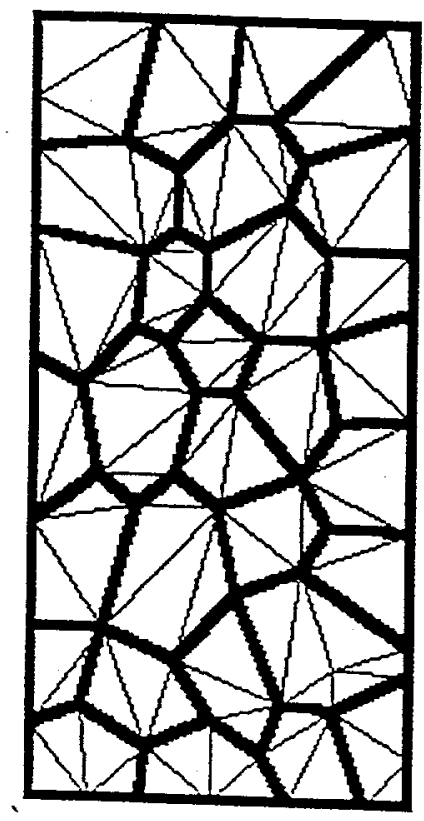



Figure $7 f$

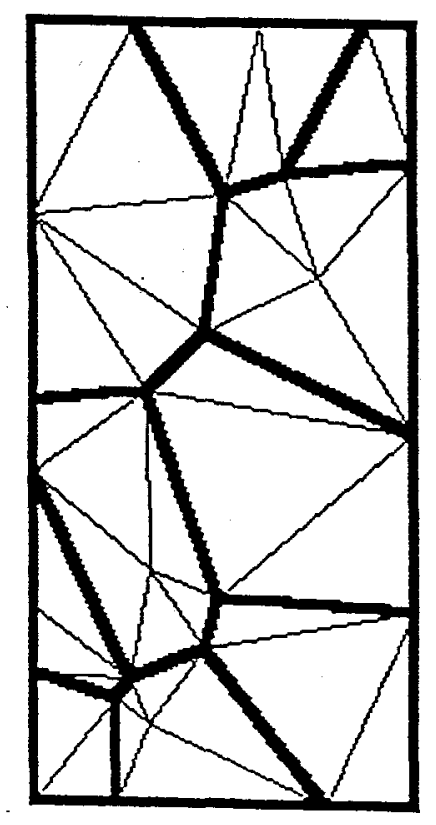


Figure $7 \mathrm{~g}$

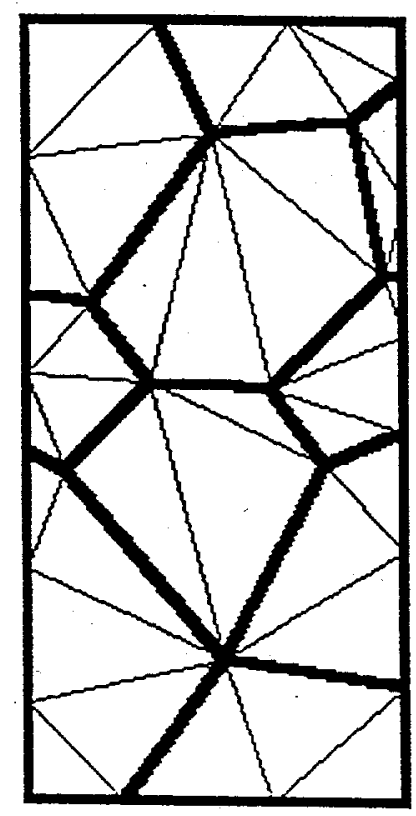


Figure $7 \mathrm{~h}$

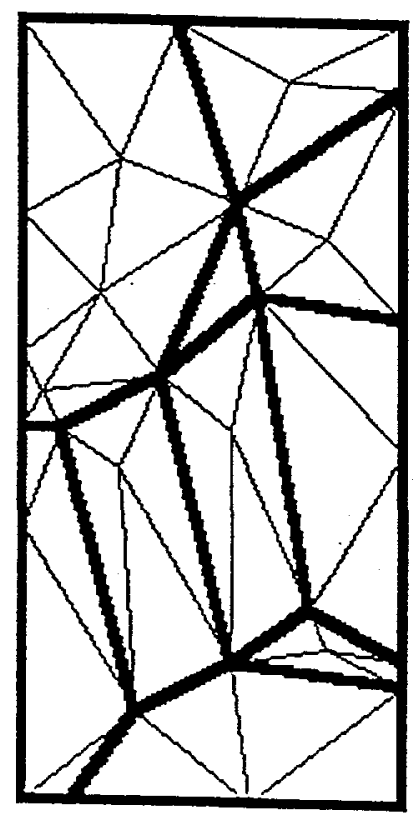


Figure 7i

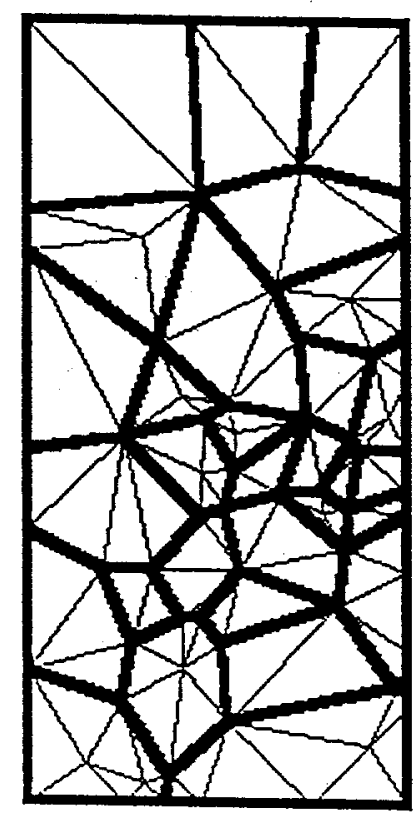


Figure 7j

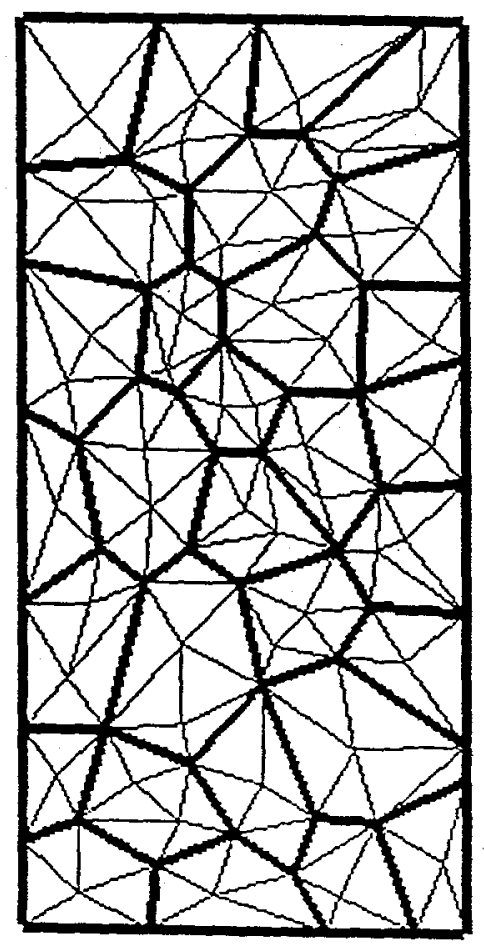


Figure $7 \mathrm{k}$

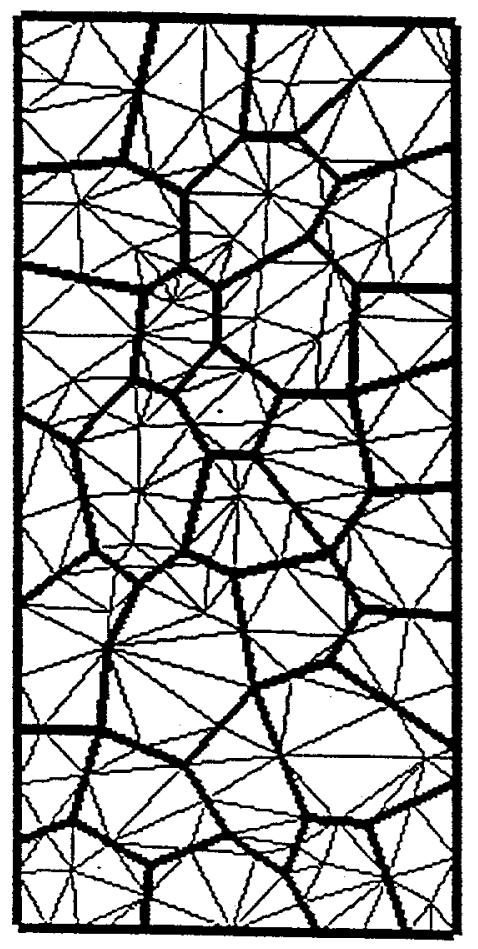


Figure 71

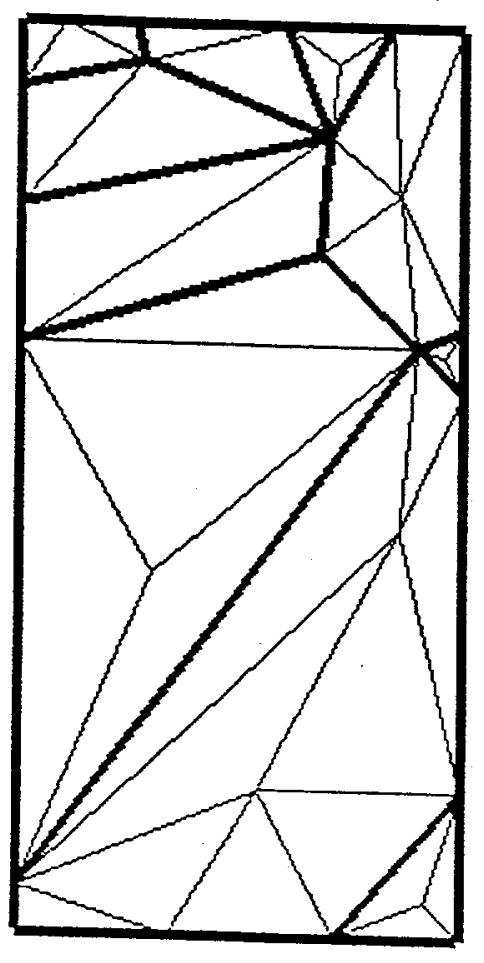


Figure $7 \mathrm{~m}$

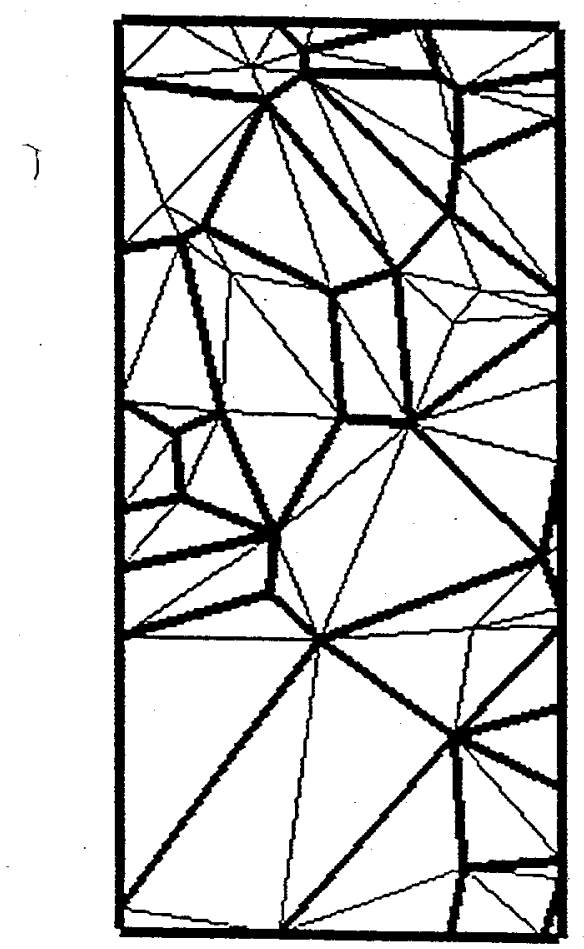


Figure 10

\section{Predicted force vs. displacement (at free end) by average grain size}

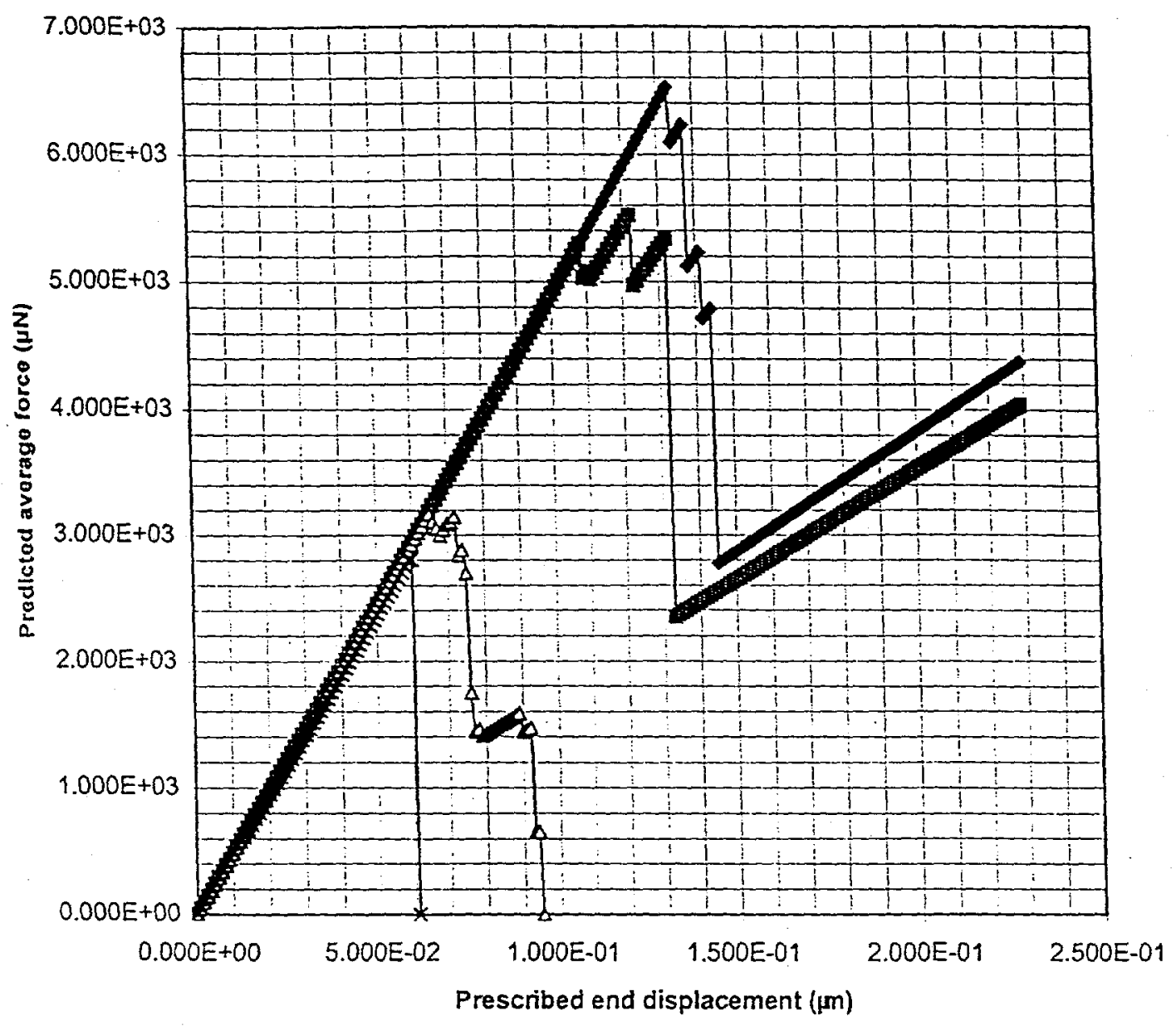

$\rightarrow$ Average grain size $=160($ Mesh 1$)$

- - Average grain size $=80$ (Mesh 2)

$\triangle$-Average grain size $=26.66($ Mesh 3$)$ :

$\rightarrow$ Average grain size $=8($ Mesh 4$)$ 


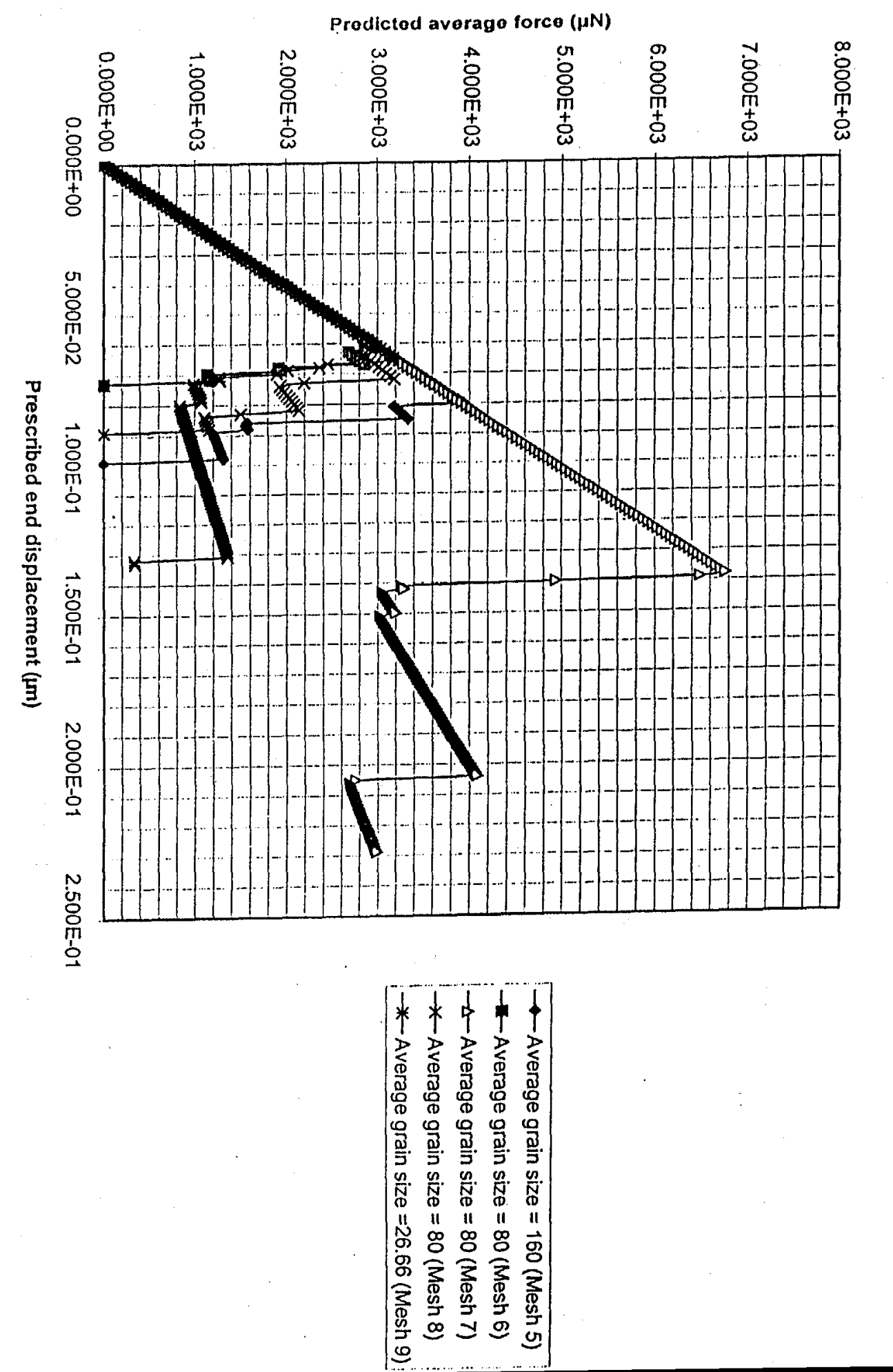

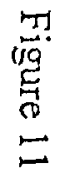

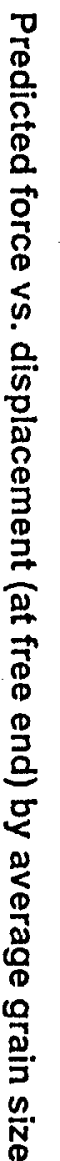


Figure 12

Predicted force vs. displacement (at free end) for mesh refinement of $\mathbf{3 0}$ grain model

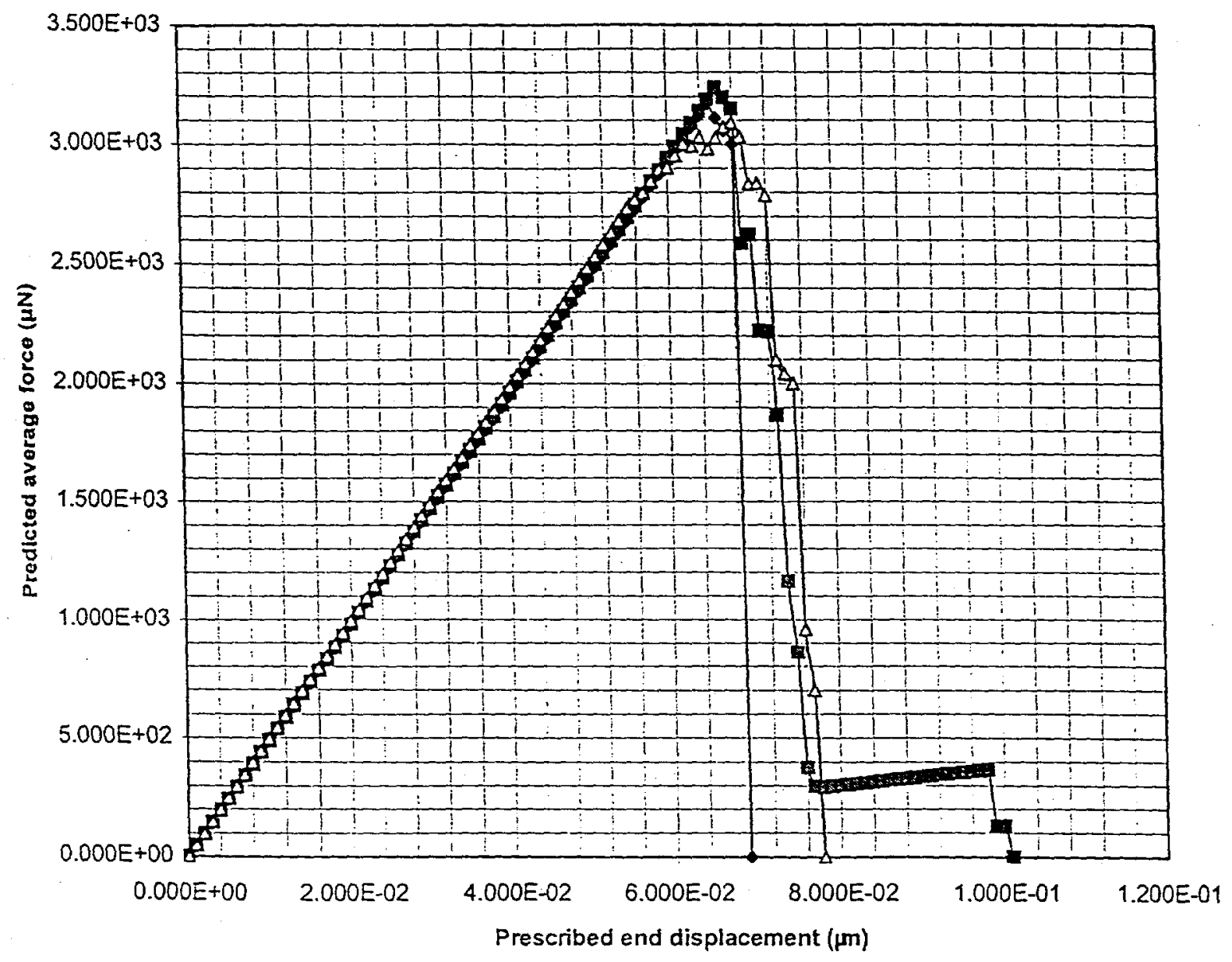

-97 elements (Mesh 3)

$\rightarrow-185$ elements (Mesh 10):

$-1-290$ elements (Mesh 11) 
Figure 13

Predicted force vs. displacement (at free end) by TRVE size

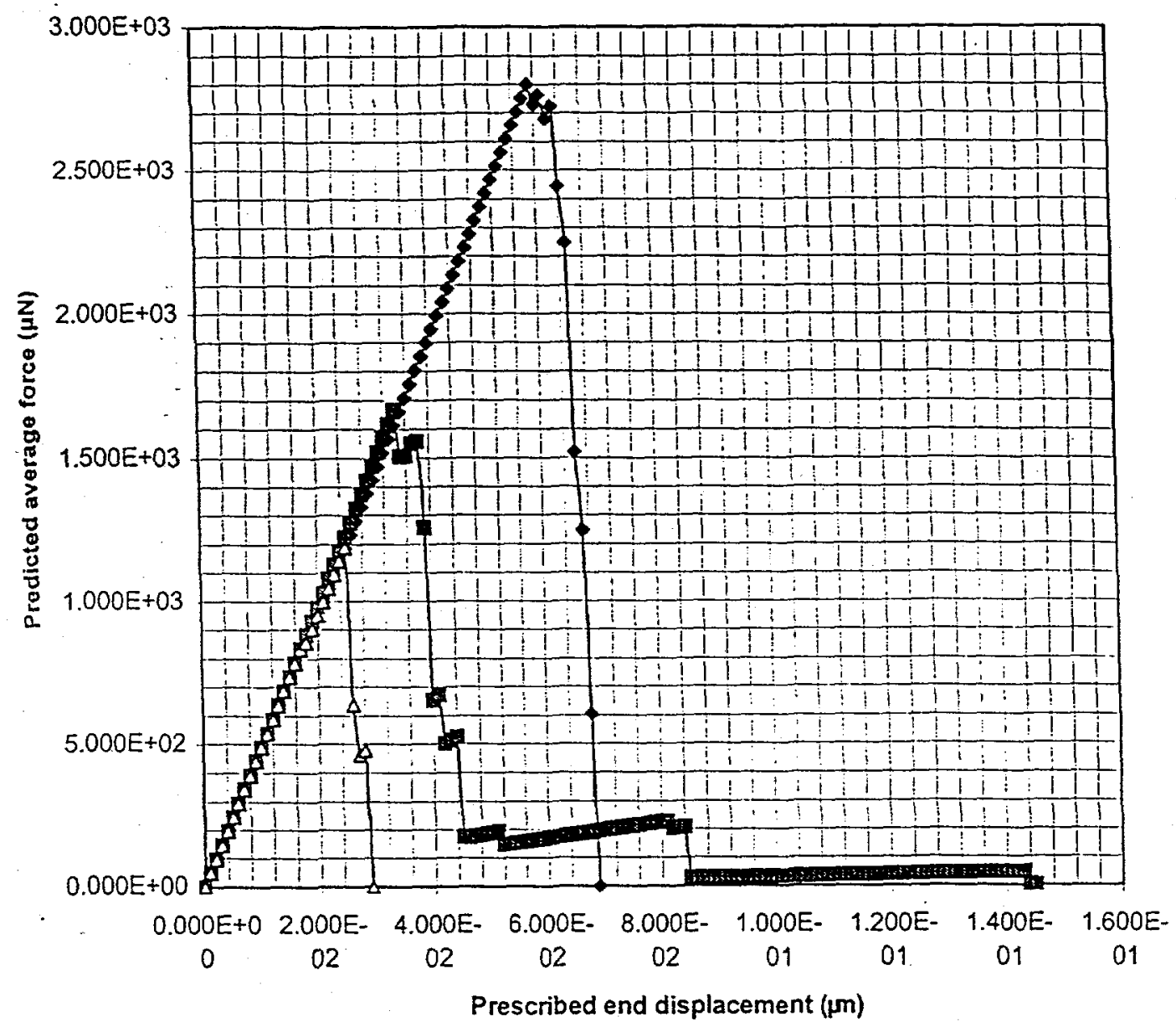

- TRVE size $=20 \mu \mathrm{m} \times 40 \mathrm{~m}($ Mesh

$\rightarrow$ TRVE size $=10 \mu \mathrm{m} \times 20 \mathrm{~m}$ (Mesh $\triangle-$ TRVE size $=5$ m mx10 m $($ Mesh 1 
Figure 14

Predicted force vs. displacement (at free end) for 10 grain models by geometry

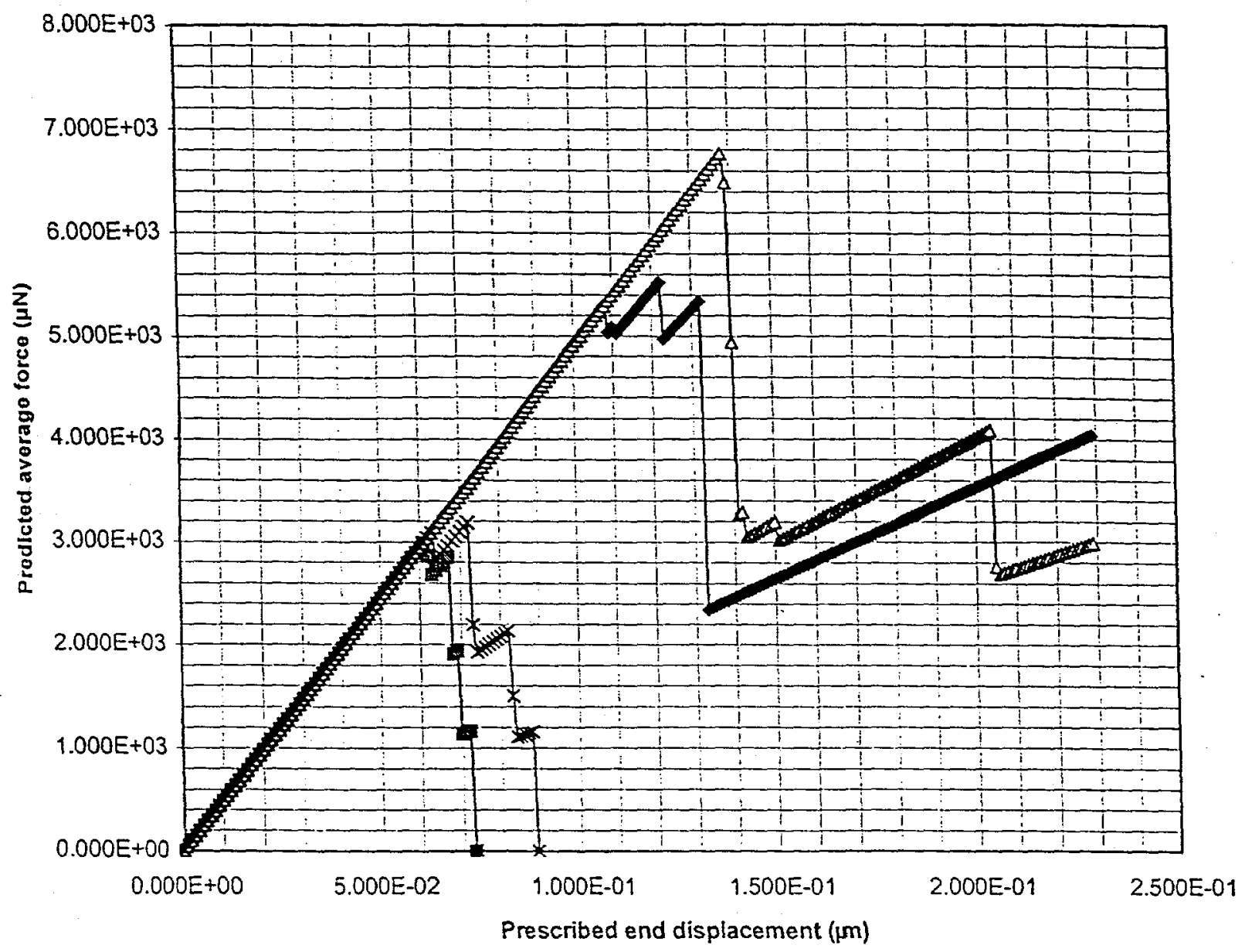

$\multimap$ Geometry 3 (Mesh 2)

$\rightarrow$ - Geometry 4 (Mesh 6) - - Geometry 5 (Mesh 7) $\rightarrow$ Geometry 6 (Mesh 8 ) 
Figure 9

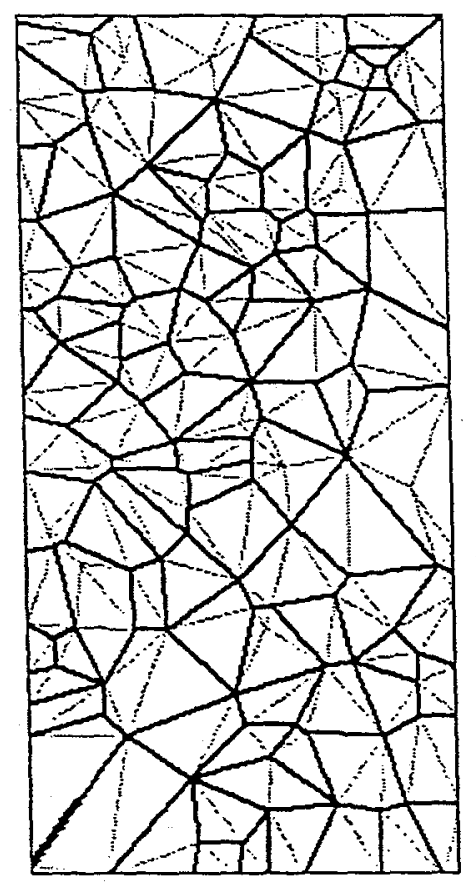

TimeElapsed: 00:13

Debonds: 1

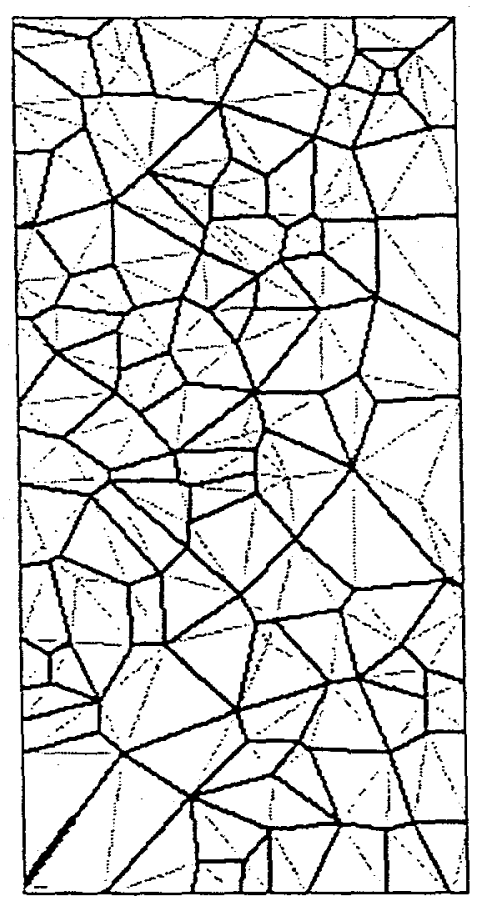

TimeElapsed: 00:57

Debonds: 3

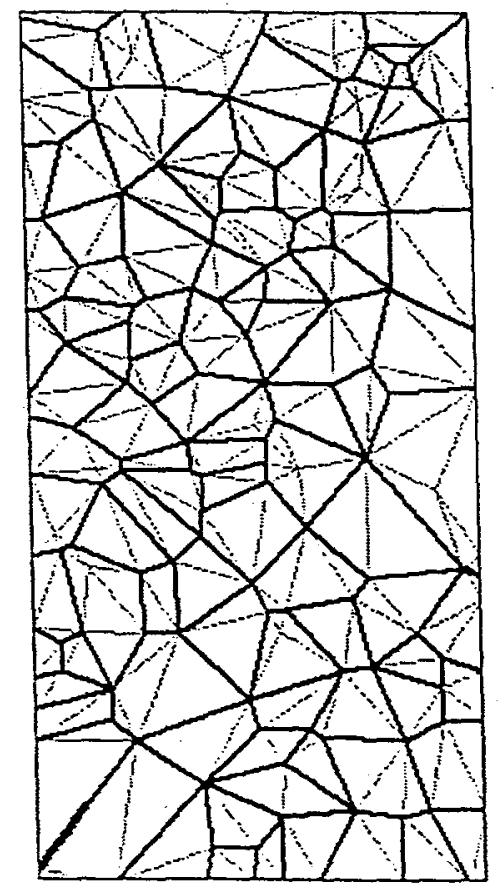

TimeElapsed: 00:55

Debonds: 2

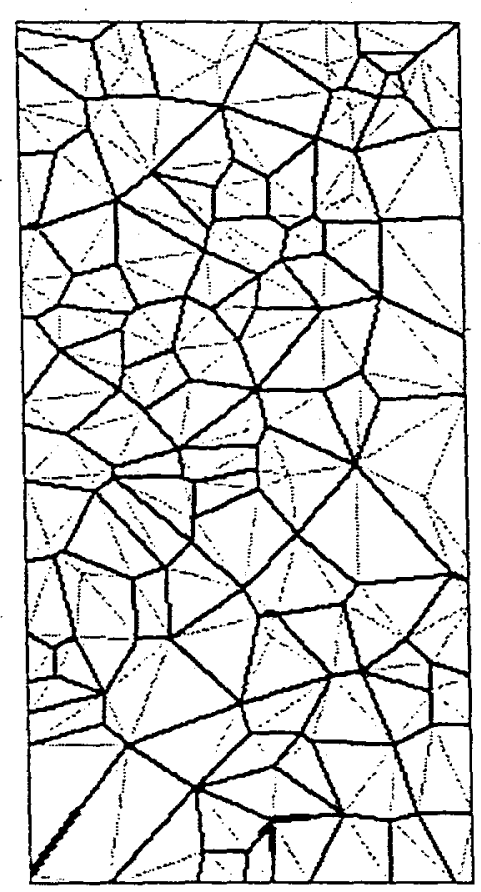

TimeElapsed: 01:00

Debonds: 7 
Figure 8

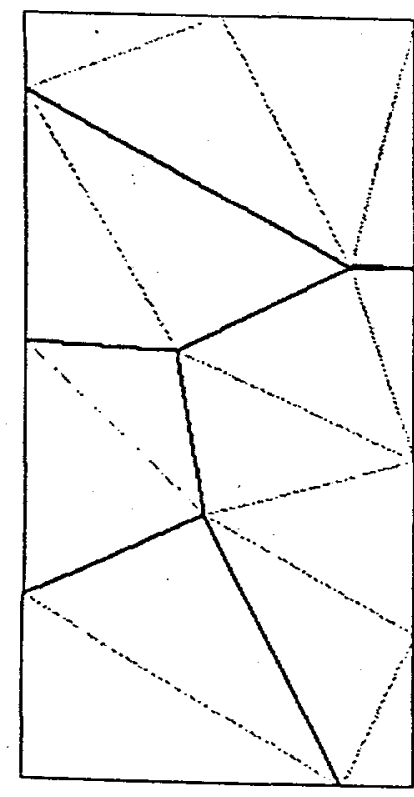

TimeElapsed: 02:13

Debonds: 1

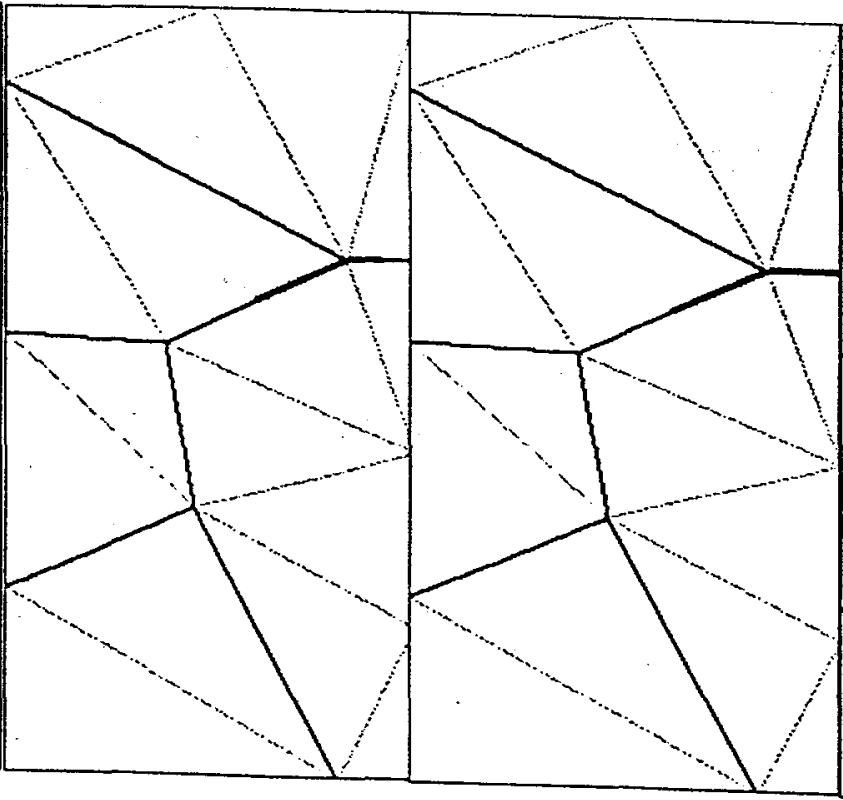

TimeElapsed: 02:14

Debonds: 2
TimeElapsed: 02:19

Debonds: 3

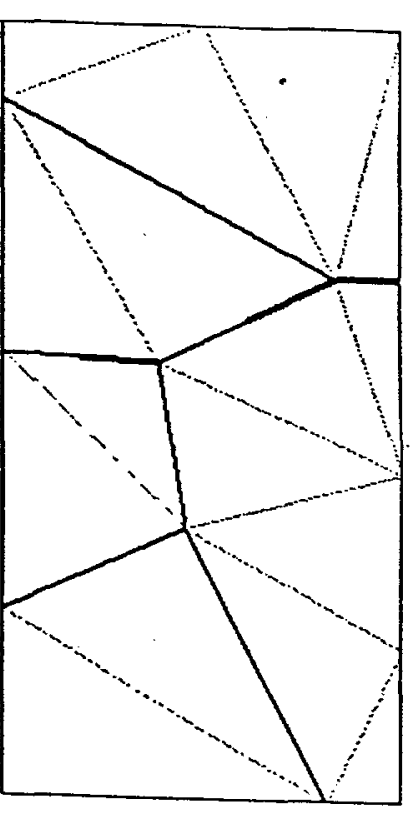

TimeElapsed: 02:22

Debonds: 4

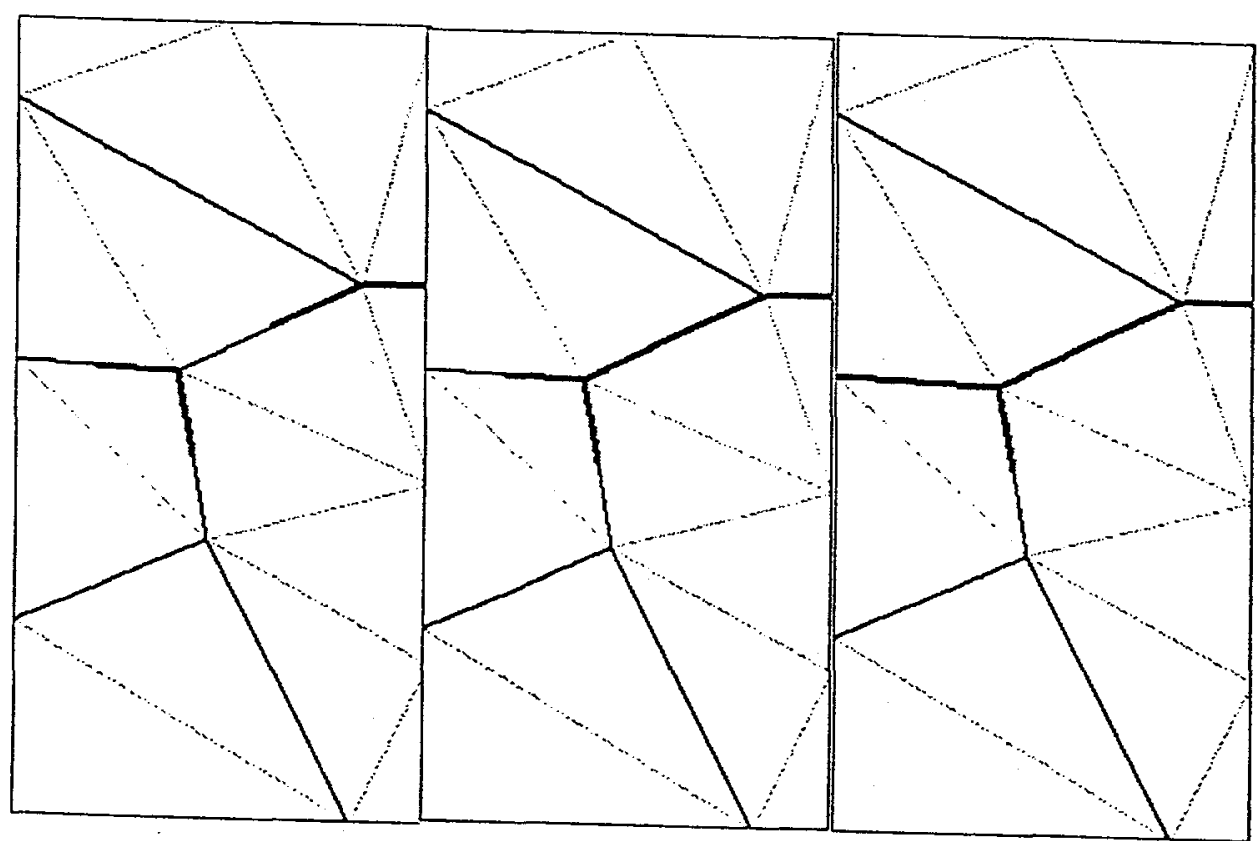

TimeElapsed: 02:23

Debonds: 5
TimeElapsed: 02:26

Debonds: 6
TimeElapsed: 03:50

Debonds: 7 
Table 1

\begin{tabular}{|c|c|c|c|c|}
\hline \multicolumn{5}{|c|}{ Geometries Considered } \\
\hline$\#$ & Geometry based on & TRVE size & \# of grains & Average 2-D Grain Size \\
\hline 1 & Voronoi tessellation & $20 \times 40$ & 5 & 160 \\
\hline 2 & Voronoi tessellation & $20 \times 40$ & 5 & 160 \\
\hline 3 & Voronoi tessellation & $20 \times 40$ & 10 & 80 \\
\hline 4 & Voronoi tessellation & $20 \times 40$ & 10 & 80 \\
\hline 5 & Voronoi tessellation & $20 \times 40$ & 10 & 80 \\
\hline 6 & Voronoi tessellation & $20 \times 40$ & 10 & 80 \\
\hline 7 & part of Geometry 11 & $5 \times 10$ & 10 & 8 \\
\hline 8 & Voronoi tessellation & $20 \times 40$ & 30 & 26.67 \\
\hline 9 & Voronoi tessellation & $20 \times 40$ & 30 & 26.67 \\
\hline 10 & part of Geometry 11 & $10 \times 20$ & 30 & 8 \\
\hline 11 & Voronoi tessellation & $20 \times 40$ & 100 & 8 \\
\hline
\end{tabular}


Table 2

\begin{tabular}{|c|c|c|c|c|}
\hline \multicolumn{5}{|c|}{ Meshes Considered } \\
\hline \# & Geometry & \# of grains & \# of elements & Average \# elementsigrain \\
\hline 1 & 1 & 5 & 16 & 3.2 \\
\hline 2 & 3 & 10 & 28 & 2.8 \\
\hline 3 & 8 & 30 & 97 & 3.233 \\
\hline 4 & 11 & 100 & 292 & 2.92 \\
\hline 5 & 2 & 5 & 26 & 5.2 \\
\hline 6 & 4 & 10 & 37 & 3.7 \\
\hline 7 & 5 & 10 & 37 & 3.7 \\
\hline 8 & 6 & 10 & 44 & 4.4 \\
\hline 9 & 9 & 30 & 109 & 3.633 \\
\hline 10 & 8 & 30 & 185 & 6.167 \\
\hline 11 & 8 & 30 & 290 & 9.67 \\
\hline 12 & 7 & 10 & 37 & 3.7 \\
\hline 13 & 10 & 30 & 88 & 2.93 \\
\hline
\end{tabular}


Table 3

\begin{tabular}{|c|c|}
\hline \multicolumn{2}{|c|}{ Material Properties for Timetal-21S at 482 C } \\
\hline Young's Modulus & $98100 \mathrm{MPa}$ \\
\hline Poisson's ratio & 0.34 \\
\hline Elastic Yield Stress & $90000 \mathrm{MPa}$ \\
\hline
\end{tabular}


